Journal of Mathematical Sciences and Modelling, 3 (1) (2020) 10-24
Research Article
Journal of Mathematical Sciences and Modelling
Journal Homepage: www.dergipark.gov.tr/jmsm
ISSN 2636-8692
DOI: http://dx.doi.org/10.33187/jmsm.632590

\title{
A Mathematical Note on the Evolutionary Competitiveness of the Trisexual Nematode Auanema Rhodensis
}

\author{
Florian Rupp ${ }^{1}$ \\ ${ }^{1}$ Universität der Bundeswehr, Research Center Code, and, Center of Conflict Resolution (CCR Munich), Munich, Germany
}

\author{
Article Info \\ Keywords: Auanema Rhodensis, bifur- \\ cation, Global dynamics, Mathematical \\ biology, Population dynamics, Repro- \\ duction dynamics, Stability, Trisexual \\ species. \\ 2010 AMS: $37 C 70,37 G 10,37 N 25$, \\ $92 D 25$. \\ Received: 13 October 2019 \\ Accepted: 6 April 2020 \\ Available online: 24 April 2020
}

\begin{abstract}
Trisexual species with female, male and self-fertilizing hermaphrodite sub-populations are rather exceptions in nature. Though, certain nematode/ worm species, like Auanema Rhodensis, have evolved that way. Applying Kendall-like non-logistic mating functions, we provide a series of reproduction models to holistically study the iterations between the sexes and shed light on the increased population stability/ survival strength compared to bisexual species or trisexual species with non-self-fertilizing hermaphrodites. Besides the increased survival strength, the survival of such trisexual species populations is, in contrast to usually known (bisexual) species populations, entirely linked to the relation between birth and death proportionality factors, and no population thresholds are required for survival. In that sense, while mathematically studying the complete equilibria and bifurcation landscape in terms of existence and (non-linear) stability, as well as the global dynamics of these models, we provide a comprehensive analysis of the reproduction dynamics of trisexual species.
\end{abstract}

\section{Introduction}

Recently, the trisexual nematode/ worm Auanema Rhodensis has drawn a lot of attention as an amazing example for reproductive mode evolution and, especially, for bending the typical rules of genetics, cf. Refs. [1, 2, 3, 4]. The nematode species Auanema Rhodensis is a member of the Auanema (Rhabditina) genus that evolved several trisexual species which have female, male and hermaphrodite members, cf. Ref. [5]. Auanema Rhodensis hermaphrodite's are self-fertilizing which is very uncommon in particular in the context of having a female and male sex as well. Moreover, the way Auanema Rhodensis handles the 'X'-chromosome is also not typical at all, see Ref. [4]: both the females and hermaphrodites are XX, whereas the males have a single X. The females produce eggs bearing one X-chromosome, males produce exclusively X-bearing sperms ${ }^{1}$, and hermaphrodites produce XX-bearing sperm and eggs with no X. Hence, crossing females and males leads to either female or hermaphrodite offspring, males and hermaphrodites produce only male offspring, and the self-fertilizing hermaphrodites produce either female or hermaphrodite offspring, too. These reproduction dynamics are sketched in Fig. 1.1.

In Ref. [1] it is shown that XX-individuals of Auanema Rhodensis become hermaphrodites or females depending on whether they undergo the non-feeding juvenile stage, called dauer larva, or not. The actual reasons for undergoing or skipping the dauer larva stage seem to be still unknown, cf. Ref. [4].

Typically, and in alignment with the commonly accepted Mendel-rules of genetics, females are XX (producing X-bearing eggs) and males are XY (producing an equal split of the heterogametic sperm with an X-and a Y-chromosome/ -gamet), which typically leads to an (almost) equal ratio of male and female offspring. Moreover, when it comes to hermaphrodites, which are pretty common among invertebrates, typically one observes them being XX-type as well and producing one X-bearing egg and one X-bearing sperm such that it needs two of them to create offspring.

\footnotetext{
${ }^{1}$ Though, the cell-biological mechanism of meiosis suggest that an X-male must produce two sperm cells with a haploid set of chromosomes, i.e. an X-bearing sperm together with a non-X-bearing sperm. These non-X-bearing sperms are discarded later on.
} 


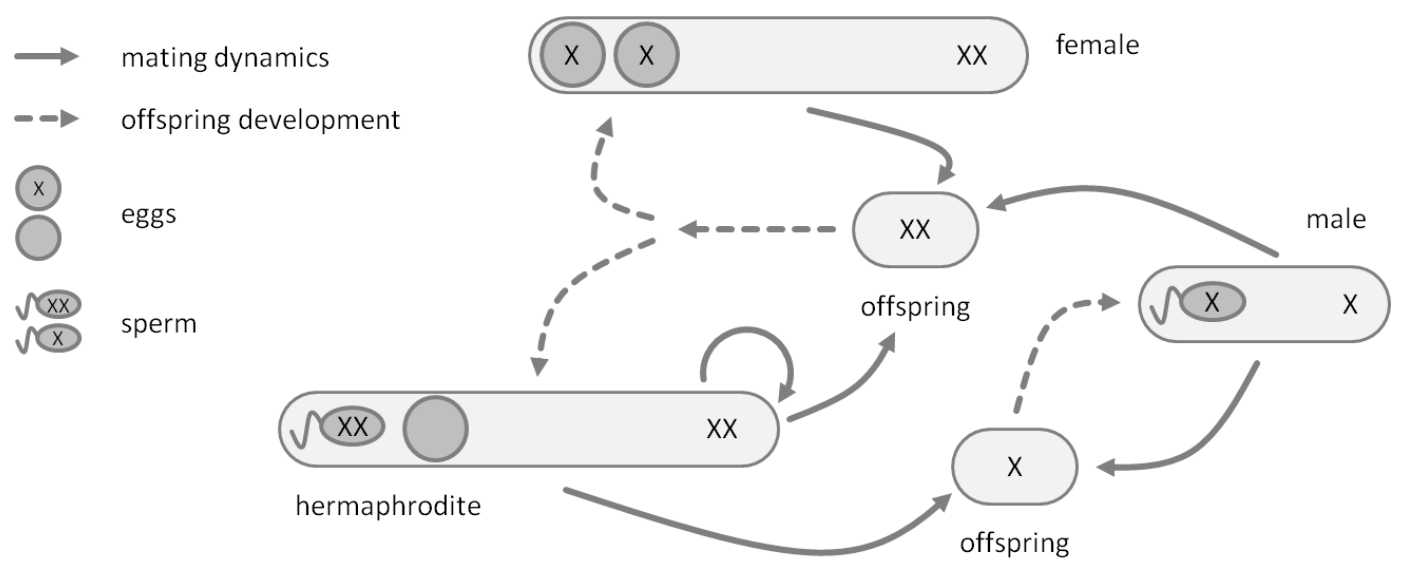

Figure 1.1: Sketch of the reproduction dynamics of the trisexual nematode Auanema Rhodensis and the interaction between the female, male and hermaphrodite species.
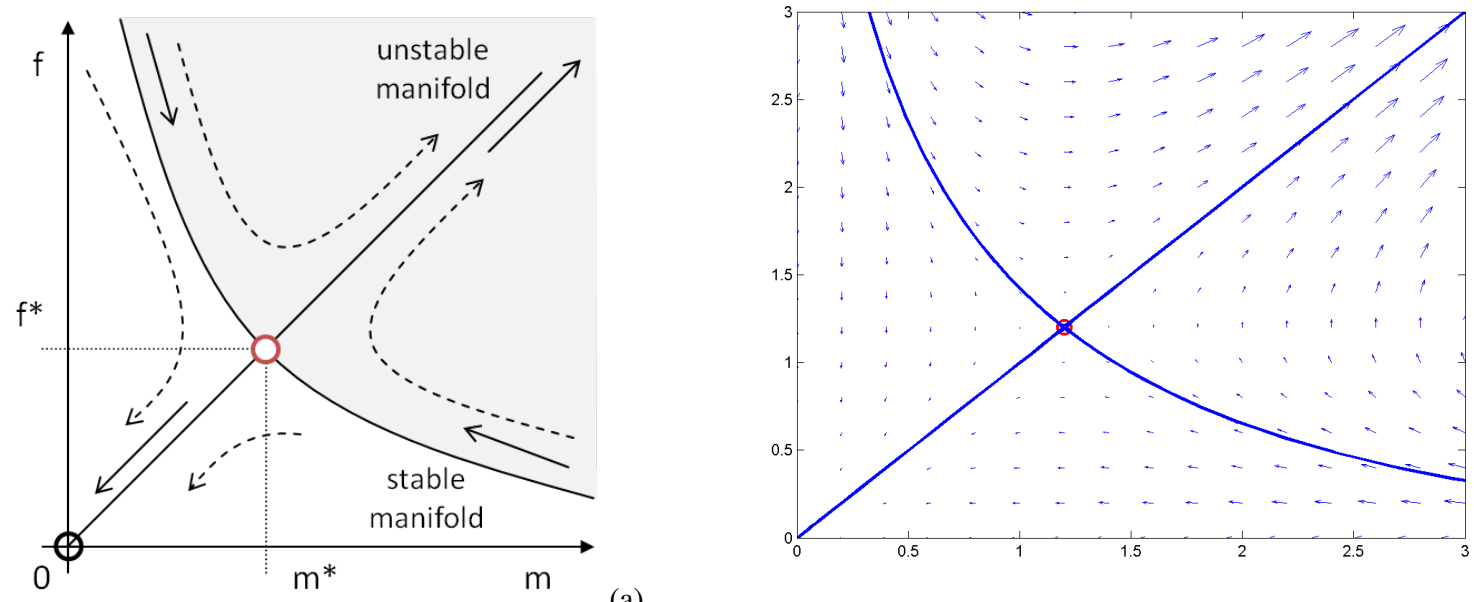

(a)

Figure 1.2: Sketch of the dynamics for a bisexual female-male population (a), and a corresponding simulation with $\alpha=0.5$ (which leads to a symmetric behavior), $\beta=1$, and $\delta=0.6(\mathrm{~b})$.

So the major question is whether the reproduction mode of Auanema Rhodensis has or has not some evolutionary competitive advantage compared to the typical reproduction mode found in non-self-fertilizing trisexual species?

Therefore, we set-up a series mathematical ordinary differential equation models for both the Auanema Rhodensis population as well as for a non-self-fertilizing trisexual population and compare the results of the corresponding mathematical discussions in terms of equilibria and invariant structures, their stability and domains of attraction. Hereby, our interest is on the early population growth dynamics to obtain insights on how fast a population can occupy a given biological niche and how few specimen are required to keep a population alive. We assume these traits (fast growth dynamics and population resilience at low numbers of individuals) as indicators of evolutionary fitness. Once the population has (nearly) occupied the habitat new effects, like competition for food or migration, take place, that are, by purpose, not considered here, as they occur due to population density and independent of how fast and resilient this population density is reached.

To get a first impression on how different the population dynamics of trisexual species will turn out to be compared to bisexual species, let us already in this introduction shortly discuss the time-evolution of a bisexual heterogametic population with XX-females and XY-males as just introduced, cf. Refs. [6, 7, 8, 9]. In accordance with the standard description of population dynamics, cf. Refs. [10, 11, 12, 13], we assume that the time-dependent female population $f: \mathbb{R}_{0}^{+} \rightarrow \mathbb{R}$ and the time-dependent male population $m: \mathbb{R}_{0}^{+} \rightarrow \mathbb{R}$ are completely mixed, that there are no spatial effects (i.e. we are in some kind of averaged picture) or limiting factors, like nutrients or overcrowding. Then, by suppressing the time arguments and using a Kendall-like non-logistic mating function, cf. Ref. [8], we obtain

$$
\dot{f}=\beta \cdot \alpha \cdot f \cdot m-\delta \cdot f, \quad \text { and } \quad \dot{m}=\beta \cdot(1-\alpha) \cdot f \cdot m-\delta \cdot m,
$$

where $\beta>0$ is proportionality factor related to birth events and taking fertility, mating success, etc. into account, $\delta>0$ is the sex-independent proportionality factor related to death events, and $\alpha \in(0,1)$ is a splitting factor of the offspring into females and males.

We rather immediately have for all admissible values of the parameters, that the non-negative quadrant with $f \geq 0$ and $m \geq 0$ is (positively and negatively) invariant, and that there are two equilibrium points: the origin $(f, m)=(0,0)$, i.e. the complete extinction point, and the point $\left(f^{*}, m^{*}\right):=\left(\frac{\delta}{\beta \cdot(1-\alpha)}, \frac{\delta}{\beta \cdot \alpha}\right)$. For all admissible values of the parameters the origin is asymptotically stable, which means that too small populations will eventually get extinct. Hence, one is typically interested in population threshold values at which the dynamic attraction of the origin is no longer present and where one can guarantee mathematical long-time survival of the complete population. The second equilibrium point $\left(f^{*}, m^{*}\right)$ turns out to be locally an unstable saddle with one stable and one unstable manifold. 


\begin{tabular}{|c|c|}
\hline \multicolumn{2}{|c|}{ Self-Fertilizing Hermaphrodites } \\
\hline Model & Description \\
\hline $\begin{array}{l}\text { Appendix } \\
\text { E1 }\end{array}$ & $\begin{array}{ll}\cdot \mathrm{f}+\mathrm{m} & \rightarrow \mathrm{f} \\
\cdot \mathrm{h}+\mathrm{m} & \rightarrow \mathrm{m} \\
\cdot \mathrm{h} & \rightarrow \mathrm{h}\end{array}$ \\
\hline A & $\begin{array}{ll}\cdot \mathrm{f}+\mathrm{m}, \mathrm{h} & \rightarrow \mathrm{f} \\
\cdot \mathrm{h}+\mathrm{m} & \rightarrow \mathrm{m} \\
\cdot \mathrm{h} & \rightarrow \mathrm{h}\end{array}$ \\
\hline C & $\begin{array}{ll}-f+m, h & \rightarrow f \\
\text { - } h+m & \rightarrow m \\
\text { - } h, f+m & \rightarrow h\end{array}$ \\
\hline $\begin{array}{l}\text { Appendix } \\
\text { F1 }\end{array}$ & $\begin{array}{ll}\cdot f+m & \rightarrow f \\
\cdot h+m & \rightarrow m \\
\cdot h, f+m & \rightarrow h\end{array}$ \\
\hline
\end{tabular}

f: females, m: males, h: hermaphrodites

\section{Non-Self-Fertilizing Hermaphrodites}

\begin{tabular}{|c|c|}
\hline Model & Description \\
\hline $\begin{array}{c}\text { Appendix } \\
\text { E2 }\end{array}$ & $\begin{array}{ll}\cdot f+m & \rightarrow f \\
\cdot-h+m & \rightarrow m \\
\cdot h+h & \rightarrow h\end{array}$ \\
\hline B & $\begin{array}{ll}\cdot f+m, h+h & \rightarrow f \\
\cdot-h+m & \rightarrow m \\
\cdot h+h & \rightarrow h\end{array}$ \\
\hline D & 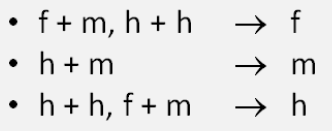 \\
\hline $\begin{array}{c}\text { Appendix } \\
\text { F2 }\end{array}$ & 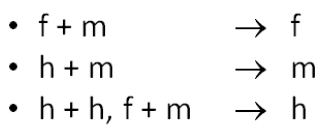 \\
\hline
\end{tabular}

Figure 1.3: Overview of the different models that we discuss in this article for trisexual species with self-fertilizing and non-self-fertilizing hermaphrodites. A chain of symbols like $f+m, h \rightarrow f$ indicates that the offspring of both the crossing of females and males and the self-fertilization of hermaphrodites evolves to females.

Fig. 1.2 gives a sketch of the phase space dynamics and some results of a numerical simulation for this bisexual population model. Besides others, it illustrates that the stable manifold of the saddle point equilibrium acts as a separatrix between the region of extinction, i.e. the domain of attraction of the origin, and the region of survival/ (unbounded) growth (the gray area in the figure). Certainly, our model does not capture the whole picture of a population, as at some time limitations to growth will be present (modeled, e.g., via logistic functions), though we see that survival of a bisexual population requires a certain threshold on the population or threshold mixture between the sexes.

Let us return to the mathematical discussion of trisexual species. In Refs. [14, 15] a heterogametic species with the three sexes XX, XY, and YY is studied. It is suggested that, under the conditions discussed there, such trisexual systems are not stable over time, and are destined to converge to bisexual systems. In Ref. [16] numerical computations, based on a stochastic evolutionary algorithm, are performed that indicated that self-fertilizing strategies are rather stable in evolutionary settings and out-competed sexual ones. Though, changing environments seem to favor bisexual species. The reproduction of the three- or pluri-/ multisexual species studied therein are such that three or more members of a species have to meet in order to produce offspring.

In this article we augment this body of knowledge by studying the very specific situation of the self-fertilizing trisexual species Auanema Rhodensis with a bisexual reproduction between females and males as well as hermaphrodites and males, and a asexual reproduction of the hermaphrodites. Moreover, as already stated, we compare this situation with that of a generic trisexual species, where, instead of a asexual reproduction, two hermaphrodites are required to cross.

In particular, we analyze several three-dimensional models, cf. Fig. 1.3: The first one is a model for Auanema Rhodensis with its selffertilizing hermaphrodites (Model A), and the second one is a model for a trisexual species with non-self-fertilizing hermaphrodites (Model B). Next, the option of female-male offspring to mature to hermaphrodites is examined. Model C applies the Auanema Rhodensis case with its self-fertilizing hermaphrodites, and Model C the case of a trisexual species with non-self-fertilizing hermaphrodites. Certain special cases are finally discussed in the appendices, like the situation that female-male offspring evolves to females and hermaphrodite offspring evolves to hermaphrodites only (Models E1 and E2), as well as both a part of female-male offspring and the whole of hermaphrodite offspring evolves to hermaphrodites and the second part of female-male offspring evolves to females (Models F1 and F2). In all these models the origin is an equilibrium point and for some parameter vales it is even unstable with a certain bifurcation scenario in Model A. Moreover, for specific values of the parameters a line of additional equilibria in the interior of the positive orthant exists.

Our key insights are that, compared to bisexual species populations, the introduction of self-fertilizing hermaphrodite members stabilizes a trisexual population. Moreover, instead of species thresholds for the female and male sub-populations, the survival of such a trisexual species populations is entirely linked to the relation between birth and death proportionality factors. I.e., no matter how small the population may become recovery is certain, provided the birth and death proportionality factors are favorable. (In contrast, a too small bi-sexual species population will for sure become extinct.)

The remainder of this article is structured as follows: In Section 2 we set-up a deterministic Model of the population dynamics of Auanema Rhodensis (Model A) and give a complete description of its equilibria in terms of existence and (non-linear) stability, as well as a description of the global dynamics of this model. Analogously, Section 3 mathematically studies Model B, Section 4 examines Model C, and Model D is analyzed in Section 5. The mathematical discussion of the somewhat restricted models A and B before that of the complete models $\mathrm{C}$ and $\mathrm{D}$ allows us to describe and develop the (standard) methods necessary for the complete model in the easier cases and then being able to refer to the previous steps later on for the required solution steps. Thus, at these later stages, we can focus on the specific and interesting additional challenges of the complete models. Finally, the main part of the text concludes with a resume in Section 6, where we summarize and interpret our results obtained by our models and their comparison with each other and bisexual species populations. As stated, the appendices study specific special cases to complete the discussion of the reproduction dynamics of trisexual species populations. First, in appendix A, we state and analyze mathematical models for species with neither hermaphrodites-producing females nor females-producing 
hermaphrodites, and second, in appendix B, we state and analyze mathematical models for species with hermaphrodites-producing females and no-females-producing hermaphrodites.

\section{A first mathematical model for the Auanema Rhodensis population (Model A)}

In a first approach to study the competitiveness of a Auanema Rhodensis population, let us assume that there is a complete bias towards female offspring after crossing female and male members of the population, and that with some splitting factor hermaphrodite offspring are females or hermaphrodites.

Following the description of the reproductive dynamics given in the introduction, let $f: \mathbb{R}_{0}^{+} \rightarrow \mathbb{R}$ denote the time-dependent female Auanema Rhodensis population, $m: \mathbb{R}_{0}^{+} \rightarrow \mathbb{R}$ the corresponding male population, and $h: \mathbb{R}_{0}^{+} \rightarrow \mathbb{R}$ the corresponding hermaphrodite population. Assuming complete mixing and spatial homogeneity of the Auanema Rhodensis population, then, according to the standard description of population dynamics, cf. Refs. $[10,11,12,13]$, the deterministic population dynamics of Auanema Rhodensis are given by

$$
\begin{aligned}
\dot{f} & =\beta_{1} \cdot f \cdot m+(1-\alpha) \cdot \beta_{3} \cdot h-\delta \cdot f, \\
\dot{m} & =\beta_{2} \cdot h \cdot m-\delta \cdot m, \\
\dot{h} & =\alpha \cdot \beta_{3} \cdot h-\delta \cdot h,
\end{aligned}
$$

where we suppressed the time arguments of the population functions $f(t), m(t)$, and $h(t)$. Here, $\beta_{1}, \beta_{2}, \beta_{3}>0$ are proportionality factors related to birth events, $\delta>0$ is the sex-independent proportionality factor related to death events, and $\alpha \in(0,1)$ a splitting factor of the hermaphrodite offspring into females and hermaphrodites ${ }^{2}$. For further reference, we will denote this set of equation Eqs. (2.1)-(2.3) as Model A.

In view of later simulations, we see that Eq. (2.3) decouples from the system Eqs. (2.1)-(2.3) such that $h(t)=h_{0} \cdot \exp \left(\left(\alpha \cdot \beta_{3}-\delta\right) \cdot t\right)$, $t \in \mathbb{R}_{0}^{+}$, where $h_{0} \geq 0$ is the initial hermaphrodite population at the initial time $t_{0}=0$.

\subsection{A mathematical discussion of Model A's equilibrium points and their stability}

For all admissible values of the parameters, we immediately have that the non-negative orthant $\mathscr{P}_{0}^{+}:=\left\{(f, m, h) \in \mathbb{R}^{3}: f, m, h \geq 0\right\}$ is invariant, and that the complete population eventually becomes extinct for a vanishing hermaphrodite population.

Moreover, for all admissible values of the parameters, the origin $(f, m, h)=(0,0,0)$ is an equilibrium (complete extinction equilibrium), where the local linearized dynamics around the origin are determined by $\dot{x}=A_{1} x$ with $x=(f, m, h)$ and

$$
\frac{\mathrm{d}}{\mathrm{d} t}\left(\begin{array}{c}
f \\
m \\
h
\end{array}\right)=\underbrace{\left(\begin{array}{ccc}
-\delta & 0 & (1-\alpha) \cdot \beta_{3} \\
0 & -\delta & 0 \\
0 & 0 & \alpha \cdot \beta_{3}-\delta
\end{array}\right)}_{=: A_{1}}\left(\begin{array}{c}
f \\
m \\
h
\end{array}\right)+\left(\begin{array}{c}
\beta_{1} \cdot f \cdot m \\
\beta_{2} \cdot h \cdot m \\
0
\end{array}\right) .
$$

Due to the triangular structure of $A_{1}$ the eigenvalues of $A_{1}$ can be read off as $-\delta<0$ with algebraic multiplicity two and corresponding 2 -dimensional eigenspace spanned by $(1,0,0)$ and $(0,1,0)$, as well as $\alpha \cdot \beta_{3}-\delta$ with the corresponding eigenspace spanned by $(1-\alpha, 0, \alpha)$. Next, the Theorem of Linearized Stability implies the following stability properties of the origin in the complete system Eqs. (2.1)-(2.3): First, for $\alpha \cdot \beta_{3}-\delta<0$ the origin is an asymptotically stable equilibrium, second, for $\alpha \cdot \beta_{3}-\delta>0$ the origin is a unstable equilibrium. Finally, taking the non-linear structure into account, we have that for $\alpha \beta_{3}-\delta=0$ the origin is a Lyapunov stable (though not asymptotically stable) hyperbolic equilibrium of the complete system Eqs. (2.1)-(2.3).

Hence, at $\alpha \cdot \beta_{3}-\delta=0$ a bifurcation takes place. Biologically, this bifurcation point corresponds to the situation where the hermaphrodite populations remains constant for all times (as the right-hand side of Eq. (2.2) vanishes). Moreover, for parameters that allow $\alpha \cdot \beta_{3}-\delta>0$ a survival of the population is guaranteed for all initial conditions with $h_{0}>0$ due to the unstable/ repulsive character of the origin and its unstable direction $(1-\alpha, 0, \alpha)$, as we will see in more detail in Sections 2.2 and 2.3.

Besides the origin, for parameters with $\alpha \cdot \beta_{3}=\delta$, i.e. at the bifurcation point, every point of the form $(f, m, h)=\left(f_{0}, 0, h_{i}\right)$ is an equilibrium as well, where $f_{0}:=f_{0}\left(h_{i}\right):=(1-\alpha) \cdot \beta_{3} \cdot h_{i} \cdot \delta^{-1}$, and $h_{i} \geq 0$ is a feasible initial value of Eq. (2.3) which reduces in the case of $\alpha \cdot \beta_{3}=\delta$ to $\dot{h}=0$. This gives rise to a line of equilibria

$$
\Gamma_{0}:=\left\{\left(f_{0}, 0, h_{i}\right) \in \mathscr{P}_{0}^{+}: f_{0}=f_{0}\left(h_{i}\right)=(1-\alpha) \cdot \beta_{3} \cdot h_{i} \cdot \delta^{-1}, h_{i}>0\right\}
$$

at the $\{m=0\}$-face of the non-negative orthant $\mathscr{P}_{0}^{+}$that emerges from the origin.

In order to determine the stability of a member $\left(f_{0}, 0, h_{i}\right) \in \Gamma_{0}$, we translate the coordinate system into $\left(f_{0}, 0, h_{i}\right)$, i.e. $(f, m, h) \mapsto$ $\left(f+f_{0}, m, h+h_{i}\right)$, and discuss the Taylor-expansion of the appropriately modified complete system Eqs. (2.1)-(2.3) about this new coordinate frame origin. Here, the correspondingly transformed equivalent dynamics read

$$
\frac{\mathrm{d}}{\mathrm{d} t}\left(\begin{array}{c}
f \\
m \\
h
\end{array}\right)=\underbrace{\left(\begin{array}{ccc}
-\delta & \beta_{1} \cdot f_{0} & (1-\alpha) \cdot \beta_{3} \\
0 & \beta_{2} \cdot h_{i}-\delta & 0 \\
0 & 0 & 0
\end{array}\right)}_{=: B_{1}}\left(\begin{array}{c}
f \\
m \\
h
\end{array}\right)+\left(\begin{array}{c}
\beta_{1} \cdot f \cdot m \\
\beta_{2} \cdot h \cdot m \\
0
\end{array}\right) .
$$

\footnotetext{
${ }^{2}$ According to the description of Auanema Rhodensis hermaphrodites as being similar to the females of the species in the biological literature, cf. [1, 2, 5, 3, 4], there seems to be pure self-fertilization of the hermaphrodites only, i.e. two hermaphrodites of this species do not match. In other words, biological insights suggest that hermaphrodites of the Auanema Rhodensis species have the same sexual organs as females of this species.
} 


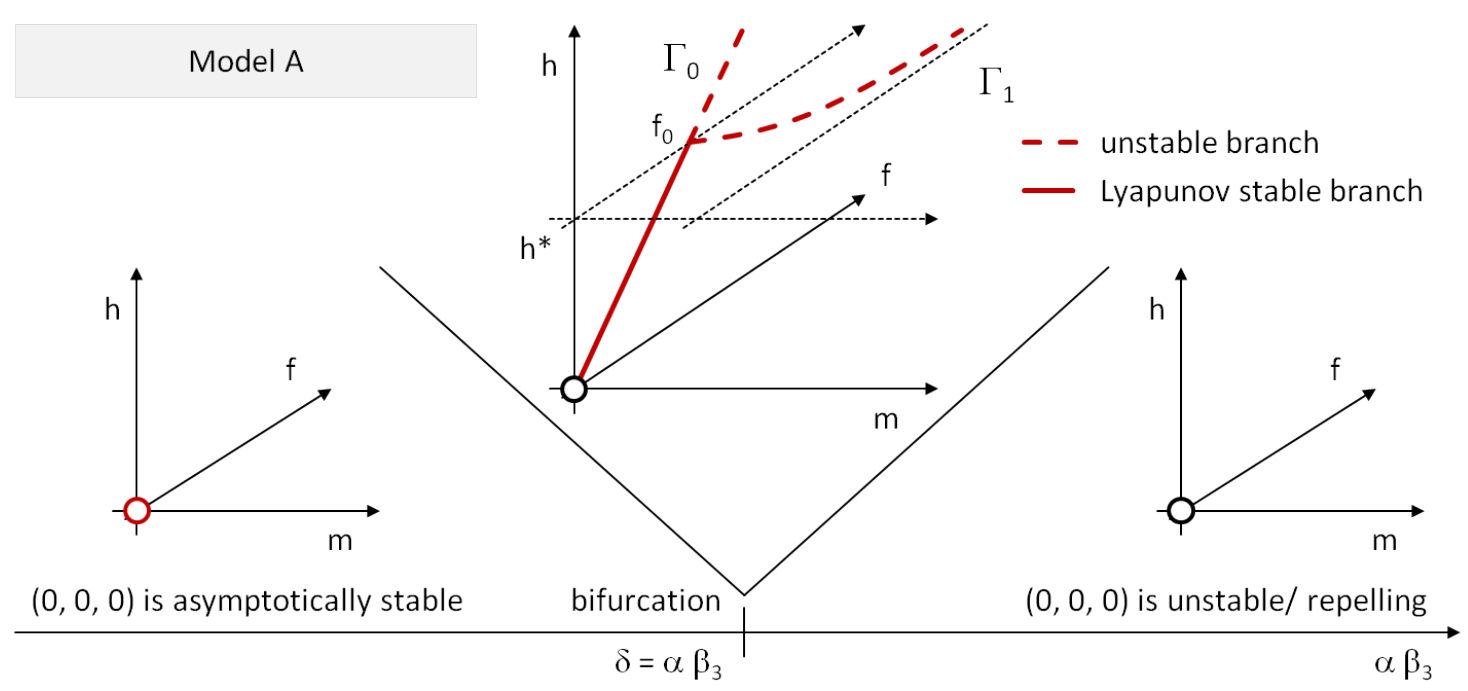

Figure 2.1: Sketch of the stability bifurcation of the extinction equilibrium at the origin and the occurrence of the lines $\Gamma_{0}$ and $\Gamma_{1}$ of additional equilibria at the bifurcation point $\alpha \cdot \beta_{3}^{-1}=\delta$.

The eigenvalues of $B_{1}$ are 0 with the corresponding eigenspace being spanned by $((1-\alpha), 0, \delta)$, and $-\delta<0$ with the corresponding eigenspace being spanned by $(1,0,0)$, as well as

$$
\beta_{2} \cdot h_{i}-\delta \begin{cases}<0, & \text { for } h_{i}<h^{*}, \\ =0, & \text { for } h_{i}=h^{*}:=\delta \cdot \beta_{2}^{-1}=\alpha \cdot \beta_{3} \cdot \beta_{2}^{-1}, \\ >0, & \text { for } h_{i}>h^{*}\end{cases}
$$

with the corresponding eigenspace being spanned by $\left(\beta_{1} \cdot f_{0}, \beta_{2} \cdot h_{i}, 0\right)$. Hence, the elements of $\Gamma_{0}$ are Lyapunov stable equilibrium points for $0 \leq h_{i}<h^{*}$ and unstable ones for $h^{*} \leq h_{i}$.

Moreover, there are additional equilibrium values $\left(f^{*}, m^{*}, h^{*}\right)$ in the interior of $\mathscr{P}_{0}^{+}$only if the parameters are such that $\alpha \cdot \beta_{3}=\delta$, i.e. again at the bifurcation point. From Eq. (2.2) we obtain $h^{*}=\delta \cdot \beta_{2}^{-1}=\alpha \cdot \beta_{3} \cdot \beta_{2}^{-1}$. Next, from Eq. (2.1) we get, after some small algebraic manipulations ${ }^{3}$,

$$
0<m^{*}=\frac{\alpha \cdot \beta_{3}}{\beta_{1} \cdot \beta_{2} \cdot f^{*}} \cdot\left(\beta_{2} \cdot f^{*}-(1-\alpha) \cdot \beta_{3}\right) \stackrel{f^{*} \rightarrow \infty}{\longrightarrow} \alpha \cdot \beta_{3} \cdot \beta_{1}^{-1}=: m_{\max }
$$

and $f^{*}>f_{0}=f_{0}\left(h^{*}\right)=(1-\alpha) \cdot \beta_{3} \cdot \beta_{2}^{-1}$. In particular, these additional equilibria $\left(f^{*}, m^{*}, h^{*}\right)$ thus form a one-dimensional family $\Gamma_{1}$ depending on the feasible values of $f^{*}$.

In order to determine the stability of a member $\left(f^{*}, m^{*}, h^{*}\right) \in \Gamma_{1}$, we progress as outlined previously for the members of $\Gamma_{0}$ : We translate the coordinate system into $\left(f^{*}, m^{*}, h^{*}\right)$, i.e. $(f, m, h) \mapsto\left(f+f^{*}, m+m^{*}, h+h^{*}\right)$, and discuss the Taylor-expansion of the appropriately modified complete system Eqs. (2.1)-(2.3) about this new coordinate frame origin. Here, the correspondingly transformed equivalent dynamics read

$$
\frac{\mathrm{d}}{\mathrm{d} t}\left(\begin{array}{c}
f \\
m \\
h
\end{array}\right)=\underbrace{\left(\begin{array}{ccc}
\beta_{1} \cdot m^{*}-\delta & \beta_{1} \cdot f^{*} & (1-\alpha) \cdot \beta_{3} \\
0 & 0 & \beta_{2} \cdot m^{*} \\
0 & 0 & 0
\end{array}\right)}_{=: C_{1}}\left(\begin{array}{c}
f \\
m \\
h
\end{array}\right)+\left(\begin{array}{c}
\beta_{1} \cdot f \cdot m \\
\beta_{2} \cdot h \cdot m \\
0
\end{array}\right),
$$

where

$$
\begin{aligned}
\beta_{1} \cdot m^{*}-\delta & =\beta_{1} \cdot \frac{\alpha \cdot \beta_{3}}{\beta_{1} \cdot \beta_{2} \cdot f^{*}} \cdot\left(\beta_{2} \cdot f^{*}-(1-\alpha) \cdot \beta_{3}\right)-\alpha \cdot \beta_{3} \\
& =\frac{\alpha \cdot \beta_{3}}{\beta_{2} \cdot f^{*}} \cdot\left(\beta_{2} \cdot f^{*}-(1-\alpha) \cdot \beta_{3}-\beta_{2} \cdot f^{*}\right)<0
\end{aligned}
$$

The eigenvalues of $C_{1}$ are $\beta_{1} \cdot m^{*}-\delta<0$ with the corresponding eigenspace being spanned by $(1,0,0)$, and the algebraically two-dimensional eigenvalue 0 with $\left(-\beta_{1} \cdot f^{*}, \beta_{1} \cdot m^{*}-\delta, 0\right)$ spanning the corresponding geometrically one-dimensional eigenspace. Taking the non-linear terms into account, we hence have that each element $\left(f^{*}, m^{*}, h^{*}\right) \in \Gamma_{1}$ is an unstable hyperbolic equilibrium of the complete system Eqs. (2.1)-(2.3).

Note, that further equilibria of Eqs. (2.1)-(2.3) do not exist and we have established a complete classification of the equilibria of our system including their stability. Fig. 2.1 summarizes our results on the types and stability properties of these equilibria as well as the underlying bifurcation scenarios.

\subsection{The global dynamics at the bifurcation point}

Once knowing what happens locally around the equilibria $(0,0,0),\left(f_{0}, 0, h_{i}\right) \in \Gamma_{0}$, and $\left(f^{*}, m^{*}, h^{*}\right) \in \Gamma_{1}$, we are interested in the global dynamics, i.e. the complete picture of the dynamics in the phase space $\mathscr{P}_{0}^{+}$. Let us start with parameters leading to the origin's bifurcation

\footnotetext{
${ }^{3}$ By virtue of Eq. (2.1), $f \neq 0$ holds at an equilibrium other than the origin.
} 

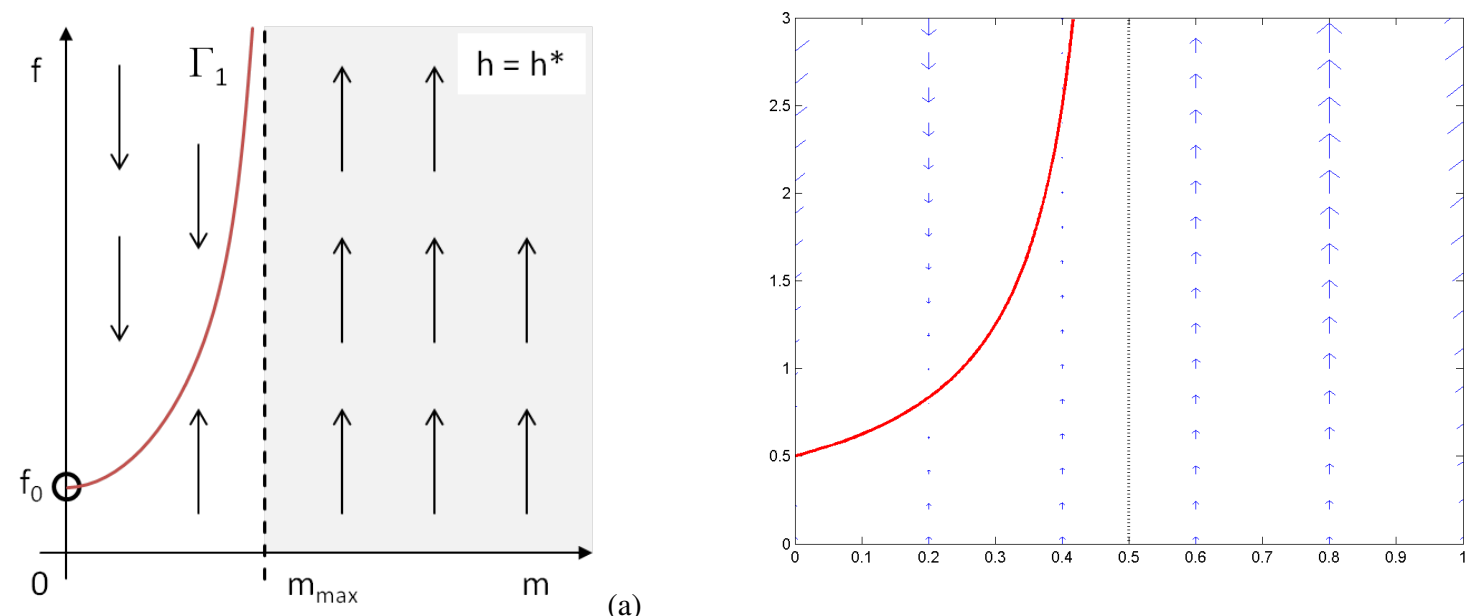

(a)

Figure 2.2: Sketch of the dynamics of Model A at the bifurcation parameters $\alpha \cdot \beta_{3}=\delta$ with $h \equiv h^{*}$ (a), and a corresponding simulation with $\alpha=0.5$, $\beta_{1}=\beta_{2}=\beta_{3}=1$, and $\delta=0.5$ (b).

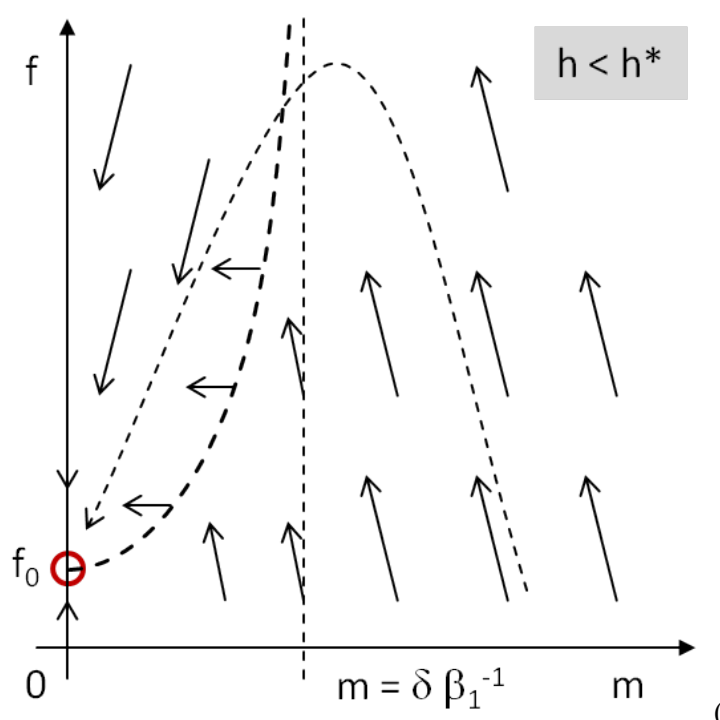

(a)

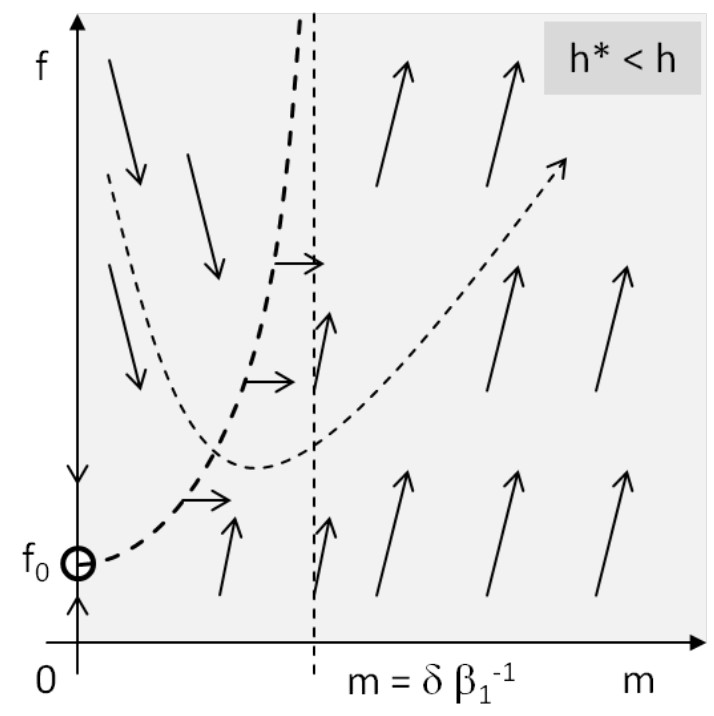

(b)

Figure 2.3: Sketch of the dynamics of Model A at the bifurcation parameters $\alpha \cdot \beta_{3}=\delta$ with $h<h^{*}$ (a), and $h>h^{*}$ (b).

point $\alpha \cdot \beta_{3}=\delta$, and discuss $f$-m-planar slices with different constant values of $h=$ const. For $\alpha \cdot \beta_{3}=\delta$ the governing system Eqs. (2.1)-(2.3) reduces to

$$
\begin{aligned}
\dot{f}(t) & =\beta_{1} \cdot f(t) \cdot m(t)+(1-\alpha) \cdot \beta_{3} \cdot h_{i}-\delta \cdot f(t), \\
\dot{m}(t) & =\left(\beta_{2} \cdot h_{i}-\delta\right) \cdot m(t),
\end{aligned}
$$

together with $\dot{h}(t)=0$, where $f(0)=f_{i} \geq 0, m(0)=m_{i} \geq 0$, and $h(0)=h_{i} \geq 0$. Here, Eq. (2.5) becomes $\dot{m}(t)=0$ (i.e. $\left.m(t)=m_{i}\right)$ for $h_{i}=h^{*}=\delta \cdot \beta_{2}^{-1}=\alpha \cdot \beta_{3} \cdot \beta_{2}^{-1}$, such that in this case the dynamics in the $f$-m-plane are governed by vectors parallel to the $f$-axis (the $m$-value is constant) pointing to $f=0$ or to $f \rightarrow \infty$ corresponding to the sign determined in Eq. (2.4).

Next, for $h_{i}<h^{*}$ we have $\dot{m}<0$ for all times and thus that the direction field is always pointing towards $m=0$ and as well either towards $f=0, f \rightarrow \infty$ or being stationary (for $\dot{f}=0$ ) depending on the sign determined in Eq. (2.4). Finally, for $h_{i}>h^{*}$ we have $\dot{m}>0$ for all times and thus that the direction field is always pointing towards $m \rightarrow \infty$ and as well either towards $f=0, f \rightarrow \infty$ or being stationary (for $\dot{f}=0$ ) depending on the sign determined in Eq. (2.4).

As some first illustration at the bifurcation point $\alpha \cdot \beta_{3}=\delta$, let us assume (i) that $\alpha=0.5$, i.e. hermaphrodites give birth to the same amount of females and hermaphrodites, and (ii) that the birth proportionality factors $\beta_{1}, \beta_{2}, \beta_{3}$ are equal. In this situation, we have $0<m^{*}<h^{*}<f^{*}$. Fig. 2.2 uses these parameters to show a sketch of the slice of the dynamics for $h \equiv h^{*}$. Here, the direction of the evolution vector field, the line of equilibria $\Gamma_{1}$, and the region of survival/ (unbounded) growth (the gray area in the figure), are included, as well as the results of a corresponding simulations that takes that actual strength of the evolution vector field into account (length of the direction vectors). Analogously, Fig. 2.3 shows sketches of the typical dynamics for $h<h^{*}$ as well as for $h^{*}<h$. Again, the region of survival/ (unbounded) growth is displayed by the gray area in the figure. Altogether this allows us to compose Fig. 2.1, Fig. 2.2, and Fig. 2.3 together and to receive the complete picture of the governing dynamics at the bifurcation point.

What remains to discuss are the dynamics for values of the parameters with $\alpha \cdot \beta_{3} \neq \delta$. 


\subsection{A global view outside of the bifurcation point}

As we have already seen, if the hermaphrodites vanish then the extinction of the complete population happens for sure. By virtue of the decoupling of the hermaphrodite dynamics from those of females and males this already gives the essential indication for survival of the complete population.

If $0<\alpha \cdot \beta_{3}<\delta$ (and $h_{i}>0$ ), then Eq. (2.3) leads to $\dot{h}(t)<0$ and hence, independent of the initial values for the females and males, the complete population will eventually get extinct. In this case, the origin is a globally asymptotically stable equilibrium governing the dynamics.

If $\delta<\alpha \cdot \beta_{3}$ (and $h_{i}>0$ ), then according to Eq. (2.3) the hermaphrodite population continues to grow (beyond any bounds) as $\dot{h}(t)>0$, i.e. we are in the setting we denoted as a survival regime. Next considering Eq. (2.2), eventually a value of $h$ is hence reached where $\beta_{2} \cdot h>\delta$, and hence eventually the size of the male population will increase (beyond any bounds) together with $h$ (presumed $m_{i}>0$ ). In complete analogy, the size of the female population will eventually increase (beyond any bounds), even if their initial size vanishes $\left(f_{i}=0\right)$.

We thus see that in Model A the survival of Auanema Rhodensis is entirely linked to the relation between the birth $\left(\alpha \cdot \beta_{3}\right)$ and death $(\delta)$ proportionality factors. This as well contrasts the dynamics of bisexual species where survival is linked to certain population thresholds and, due to the asymptotic stability of the origin, too small populations are determined to get extinct for all choices of the birth and death proportionality factors.

The results presented in Ref. [1] indicate that XX-individuals may be forced to develop into hermaphrodites instead of females (and vice versa) based on the juvenile stages they undergo. Hence, there is also some kind of (unusual) mechanism that increases the hermaphrodite population and may additionally stabilize the complete population. We will discuss this as Model C in Section 4.

Next, let us compare the our self-fertilizing hermaphrodite nematode Auanema Rhodensis (Model A) with a species that has non-selffertilizing hermaphrodite in order to discuss competitive advantages of the one or other reproduction model.

\section{Trisexual species with non-self-fertilizing hermaphrodites - Model B}

In order to gain insights into the dynamics of trisexual species, this section mathematically analyzes a model equivalent to Model A though with non-self-fertilizing hermaphrodites. I.e., two members of the hermaphrodite species are required to produce offspring such that the crossing terms with a single $h$ in Eqs. (2.1) and (2.3) are replaced by a term with $h^{2}$. Assuming again complete mixing and spatial homogeneity of the involved populations, then, according to the standard description of population dynamics, this leads to the following ordinary differential Model $\mathrm{B}^{4}$ :

$$
\begin{aligned}
\dot{f} & =\beta_{1} \cdot f \cdot m+(1-\alpha) \cdot \beta_{3} \cdot h^{2}-\delta \cdot f, \\
\dot{m} & =\beta_{2} \cdot h \cdot m-\delta \cdot m, \\
\dot{h} & =\alpha \cdot \beta_{3} \cdot h^{2}-\delta \cdot h .
\end{aligned}
$$

As in Model A, we have for all admissible values of the parameters, that $\mathscr{P}_{0}^{+}$is invariant, and that the population eventually becomes extinct for vanishing numbers of hermaphrodite population. Again, the dynamics of the hermaphrodite population $\dot{h}=h \cdot\left(\alpha \cdot \beta_{3} \cdot h-\delta\right)$ decouples from the other governing equations, with a repulsive equilibrium at $h^{*}=\delta \cdot\left(\alpha \cdot \beta_{3}\right)^{-1}$ that constitutes a threshold value first of all for the hermaphrodite sub-population and hence for the total population as well.

The origin $(f, m, h)=(0,0,0) \in \mathscr{P}_{0}^{+}$is an equilibrium (complete extinction equilibrium). In this case, the local linearized dynamics around the origin are determined by $\dot{x}=A_{2} x$ with $x=(f, m, h)$ and

$$
\frac{\mathrm{d}}{\mathrm{d} t}\left(\begin{array}{c}
f \\
m \\
h
\end{array}\right)=\underbrace{\left(\begin{array}{ccc}
-\delta & 0 & 0 \\
0 & -\delta & 0 \\
0 & 0 & -\delta
\end{array}\right)}_{=: A_{2}}\left(\begin{array}{c}
f \\
m \\
h
\end{array}\right)+\left(\begin{array}{c}
\beta_{1} \cdot f \cdot m+(1-\alpha) \cdot \beta_{3} \cdot h^{2} \\
\beta_{2} \cdot h \cdot m \\
\alpha \cdot \beta_{3} \cdot h^{2}
\end{array}\right) .
$$

Hence, the origin is an asymptotically stable equilibrium in model B for all admissible parameter values. Biologically, these are somewhat bad news as it tells us that once the population numbers are too small the population will get extinct for sure.

Moreover, there is a line $\Gamma_{2} \subset \mathscr{P}_{0}^{+}$of additional equilibria $\left(f^{*}, m^{*}, h^{*}\right) \in \Gamma_{2}$. From Eq. (3.3) we effortlessly get $h^{*}=\delta \cdot\left(\alpha \cdot \beta_{3}\right)^{-1}$, and by virtue of Eq. (3.2) we obtain that the line of equilibria $\Gamma_{2}$ exists only for parameter values where $\alpha \cdot \beta_{3}=\beta_{2}$. Next, from Eq. (3.1) we get ${ }^{5}$

$$
0 \leq m^{*}=\frac{\delta}{\alpha \cdot \beta_{1} \cdot f^{*}} \cdot\left(\alpha \cdot f^{*}-(1-\alpha) \cdot h^{*}\right) \stackrel{f^{*} \rightarrow \infty}{\longrightarrow} \delta \cdot \beta_{1}^{-1}=m_{\max }
$$

where $f^{*} \geq(1-\alpha) \cdot \alpha^{-1} \cdot h^{*}$. After linearly translating the coordinate system into $\left(f^{*}, m^{*}, h^{*}\right) \in \Gamma_{2}$, the Taylor-expansion of the appropriately modified complete system Eqs. (3.1)-(3.3) about this new coordinate frame origin reads

$$
\frac{\mathrm{d}}{\mathrm{d} t}\left(\begin{array}{c}
f \\
m \\
h
\end{array}\right)=\underbrace{\left(\begin{array}{ccc}
\beta_{1} \cdot m^{*}-\delta & \beta_{1} \cdot f^{*} & 2 \cdot(1-\alpha) \cdot \beta_{3} \cdot h^{*} \\
0 & 0 & \beta_{2} \cdot m^{*} \\
0 & 0 & \delta
\end{array}\right)}_{=: B_{2}}\left(\begin{array}{c}
f \\
m \\
h
\end{array}\right)+\left(\begin{array}{c}
\beta_{1} \cdot f \cdot m+(1-\alpha) \cdot \beta_{3} \cdot h^{2} \\
\beta_{2} \cdot h \cdot m \\
\alpha \cdot \beta_{3} \cdot h^{2}
\end{array}\right) .
$$

\footnotetext{
${ }^{4}$ Here, we are discussing the situation of non-self-fertilizing hermaphrodites that can additionally pair with males in order to have a model comparable to Model A. From a biological perspective it may make sense to assume that in this situation hermaphrodites have the same sexual organs as males and females and can thus also mate with females of the species. Considering, in terms of a comparison to the biological situation witnessed at Auanema Rhodensis, the gametic structure of female eggs and hermaphrodite sperms would, in such a pairing, result in XXX-offspring, that we can discard as not fit for survival.

${ }^{5}$ By virtue of Eq. (3.1), $f \neq 0$ holds at an equilibrium other than the origin.
} 


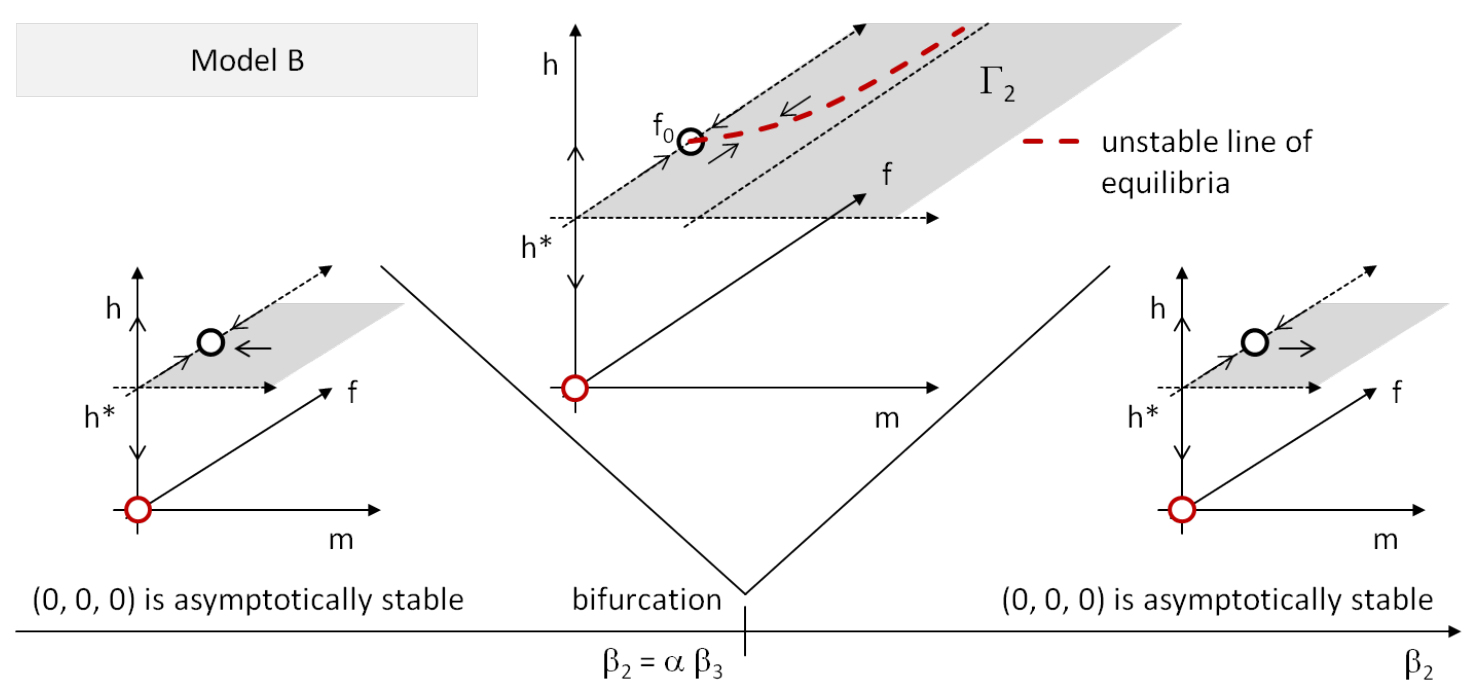

Figure 3.1: Sketch of the stability bifurcation of the equilibrium $\left(0,0, h^{*}\right)$ at and the occurrence of the line $\Gamma_{2}$ of additional equilibria at the bifurcation point $\alpha \cdot \beta_{3}=\beta_{2}$

The three distinct eigenvalues of $B_{2}$ are $\beta_{1} \cdot m^{*}-\delta<0$ with its corresponding eigenspace being spanned by $(1,0,0), 0$ with its corresponding eigenspace being spanned by $\left(\beta_{1} \cdot f^{*}, \beta_{1} \cdot m^{*}, 0\right)$, and $\delta>0$ with its corresponding eigenspace being spanned by $\left(0, \beta_{2} \cdot m^{*}, \delta\right)$. Hence, $\Gamma_{2}$ is a line of unstable (flattened) saddle point equilibria.

Let $f(0)=: f_{i}, m(0)=: m_{i}$, and $h(0)=: h_{i}$. If $\alpha \cdot \beta_{3}=\beta_{2}$ and $0<h_{i}<h^{*}$, then Eq. (3.3) leads to $\dot{h}(t)<0$ and hence, independent of the initial values for the females and males as well as the values of the other parameter, the complete population will eventually get extinct. On the other hand, if $\alpha \cdot \beta_{3}=\beta_{2}$ and $h^{*}<h_{i}$ then according to Eq. (3.3) the hermaphrodite population continues to grow (beyond any bounds) as $\dot{h}(t)>0$, i.e. we are in a survival regime. Next considering Eq. (3.1), eventually a value of $h$ is hence reached where $\beta_{2} \cdot h>\delta$, and hence eventually the size of the male population will increase (beyond any bounds) together with $h$ (presumed $m_{i}>0$ ). In complete analogy, the size of the female population will eventually increase (beyond any bounds), even if their initial size vanishes $\left(f_{i}=0\right)$. In particular, in the case $\alpha \cdot \beta_{3}=\beta_{2}$ the affine plane $h=h^{*}$ serves as a 2-dimensional separatrix between the complete extinction regime and the survival regime.

What remains is to discuss the dynamics if $\alpha \cdot \beta_{3} \neq \beta_{2}$. Let $f_{0}:=f(0), m_{0}:=m(0)$, and $h_{0}:=h(0)$ denote the initial population sizes of the female, male, and hermaphrodite sub-populations. Besides the origin, the point $(f, m, h)=\left(\frac{1-\alpha}{\alpha} \cdot h^{*}, 0, h^{*}\right)$, with $h^{*}=\delta \cdot\left(\alpha \cdot \beta_{3}\right)^{-1}$, is an equilibrium for all admissible parameter values. Due to the instability in the $h$-direction, this additional equilibrium point is unstable.

First for $\beta_{2}<\alpha \cdot \beta_{3}$, based on Eq. (3.3) if, $h_{0}<h^{*}=\delta \cdot\left(\alpha \cdot \beta_{3}\right)^{-1}$, then, irrespective of the size of the female and male sub-populations, the hermaphrodite sub-population and hence the complete population eventually gets extinct. If $h_{0}=h^{*}=\delta \cdot\left(\alpha \cdot \beta_{3}\right)^{-1}<\delta \cdot \beta_{2}^{-1}$, then the hermaphrodite sub-population stays constant for all times, i.e. $h(t) \equiv h^{*}$ for all $t \in \mathbb{R}_{0}^{1}$, whereas the male sub-population eventually gets extinct due to Eq. (3.2). Next, in this limit $h(t) \equiv h^{*}$ and $m \equiv 0$ Eq. (3.1) becomes $\dot{f}=\delta \cdot\left(\frac{(1-\alpha) \cdot \delta}{\alpha^{2} \beta_{2}}-f\right)$ such that all initial conditions $\left(f_{0}, m_{0}, h^{*}\right)$ in the affine plane $h=h^{*}$ converge towards the unstable equilibrium $(f, m, h)=\left(\frac{1-\alpha}{\alpha} \cdot h^{*}, 0, h^{*}\right)$. Finally, if $h_{0}>h^{*}=\delta \cdot\left(\alpha \cdot \beta_{3}\right)^{-1}$, then the hermaphrodite sub-population and thus both, the male and the female sub-populations will eventually, grow beyond all bounds (survival regime). Hence, in the case $\beta_{2}<\alpha \cdot \beta_{3}$ the affine plane $h=h^{*}$ with its in this plane attractive equilibrium $(f, m, h)=\left(\frac{1-\alpha}{\alpha} \cdot h^{*}, 0, h^{*}\right)$ serves as a separatrix between the complete extinction regime and the survival regime.

Second for $\beta_{2}>\alpha \cdot \beta_{3}$, we analogously conclude complete extinction if $h_{0}<h^{*}=\delta \cdot\left(\alpha \cdot \beta_{3}\right)^{-1}$. If $h_{0}=h^{*}=\delta \cdot\left(\alpha \cdot \beta_{3}\right)^{-1}>\delta \cdot \beta_{2}^{-1}$, then the hermaphrodite sub-population stays constant for all times, i.e. $h(t) \equiv h^{*}$ for all $t \in \mathbb{R}_{0}^{1}$, whereas for positive initial conditions the male subpopulation eventually grows beyond all bounds due to Eq. (3.2). Then, due to Eq. (3.1), the female sub-population grows beyond all bounds as well. For $m_{0}=0$ an initial condition $\left(f_{0}, 0, h^{*}\right)$ either is or eventually converges towards the equilibrium $(f, m, h)=\left(\frac{1-\alpha}{\alpha} \cdot h^{*}, 0, h^{*}\right)$. If $h_{0}>h^{*}=\delta \cdot\left(\alpha \cdot \beta_{3}\right)^{-1}$, then as above, we can conclude that we are again in a survival regime for all initial conditions $\left(f_{0}, m_{0}, h_{0}\right)$. Hence, in the case $\beta_{2}>\alpha \cdot \beta_{3}$ the affine plane $h=h^{*}$ serves again as a separatrix between the complete extinction regime and the survival regime.

The complete bifurcation diagram is sketched in Fig. 3.1. Note that further equilibria of Eqs. (3.1)-(3.3) do not exist and we have thus indeed established a complete classification of the equilibria of our system including their stability.

In summary we can already conclude that survival/ proliferation of the species in model A depends on the relation between the birth and death parameters. On the other hand, independent of the feasible parameter values, the non-self-fertilizing exhibits the classical feature of bi-sexual species of population thresholds. In particular, in this case, a certain number of hermaphrodites.

\section{Model C}

As a significant extension of Model A, we now include the situation that a part $1-\gamma$ with $\gamma \in(0,1)$ of the female-male offspring may become hermaphrodites. Thus, in particular Eq. 2.3 is changed by an additional term. Assuming again complete mixing and spatial homogeneity of the involved populations, then, according to the standard description of population dynamics, this leads to the following ordinary differential 
model C:

$$
\begin{aligned}
\dot{f} & =\gamma \cdot \beta_{1} \cdot f \cdot m+(1-\alpha) \cdot \beta_{3} \cdot h-\delta \cdot f, \\
\dot{m} & =\beta_{2} \cdot h \cdot m-\delta \cdot m, \\
\dot{h} & =\alpha \cdot \beta_{3} \cdot h+(1-\gamma) \cdot \beta_{1} \cdot f \cdot m-\delta \cdot h,
\end{aligned}
$$

As in our previous models, the non-negative orthant $\mathscr{P}_{0}^{+}$is invariant for all admissible values of the parameters. Contrary to the previous models, though, and as intended by introducing the transport term $(1-\gamma) \cdot \beta_{1} \cdot f \cdot m$ from female-male offspring towards the hermaphrodite sub-population, a vanishing hermaphrodite sub-population, this time, does not automatically imply extinction of the complete population.

Like in Model A, for all admissible values of the parameters, the origin $(f, m, h)=(0,0,0)$ is an equilibrium (complete extinction equilibrium), where the local linearized dynamics around the origin are determined by $\dot{x}=A_{3} x$ with $x=(f, m, h)$ and

$$
\frac{\mathrm{d}}{\mathrm{d} t}\left(\begin{array}{c}
f \\
m \\
h
\end{array}\right)=\underbrace{\left(\begin{array}{ccc}
-\delta & 0 & (1-\alpha) \cdot \beta_{3} \\
0 & -\delta & 0 \\
0 & 0 & \alpha \cdot \beta_{3}-\delta
\end{array}\right)}_{=: A_{3}}\left(\begin{array}{c}
f \\
m \\
h
\end{array}\right)+\left(\begin{array}{c}
\gamma \cdot \beta_{1} \cdot f \cdot m \\
\beta_{2} \cdot h \cdot m \\
(1-\gamma) \cdot \beta_{1} \cdot f \cdot m
\end{array}\right) .
$$

The eigenvalues of $A_{3}$ are $-\delta<0$ with algebraic multiplicity two and corresponding 2-dimensional eigenspace spanned by $(1,0,0)$ and $(0,1,0)$, as well as $\alpha \cdot \beta_{3}-\delta$ with the corresponding eigenspace spanned by $(1-\alpha, 0, \alpha)$. For $\alpha \cdot \beta_{3}-\delta<0$ the origin is an asymptotically stable equilibrium, and, due to the non-linear part, for $\alpha \cdot \beta_{3}-\delta \geq 0$ the origin is a unstable equilibrium. Again, a bifurcation w.r.t. to the stability of the origin takes place at $\alpha \cdot \beta_{3}=\delta$.

Moreover, at this bifurcation point $\alpha \cdot \beta_{3}=\delta$ the line

$$
\Gamma_{3}:=\left\{\left(f_{0}, 0, h_{i}\right) \in \mathscr{P}_{0}^{+}: f_{0}=f_{0}\left(h_{i}\right)=(1-\alpha) \cdot \beta_{3} \cdot h_{i} \cdot \delta^{-1}, h_{i}>0\right\}
$$

is (as in Model A) a line of equilibria at the $\{m=0\}$-face of $\mathscr{P}_{0}^{+}$that emerges from the origin. The correspondingly transformed equivalent dynamics about each member $\left(f_{0}, 0, h_{i}\right) \in \Gamma_{3}$ are given by

$$
\frac{\mathrm{d}}{\mathrm{d} t}\left(\begin{array}{c}
f \\
m \\
h
\end{array}\right)=\underbrace{\left(\begin{array}{ccc}
-\delta & \gamma \cdot \beta_{1} \cdot f_{0} & (1-\alpha) \cdot \beta_{3} \\
0 & \beta_{2} \cdot h_{i}-\delta & 0 \\
0 & (1-\gamma) \cdot \beta_{1} \cdot f_{0} & 0
\end{array}\right)}_{=: B_{3}}\left(\begin{array}{c}
f \\
m \\
h
\end{array}\right)+\left(\begin{array}{c}
\gamma \cdot \beta_{1} \cdot f \cdot m \\
\beta_{2} \cdot h \cdot m \\
(1-\gamma) \cdot \beta_{1} \cdot f \cdot m
\end{array}\right),
$$

and, in complete alignment to the eigenvalue structure of $B_{3}$, the eigenvalues of the rank two matrix $B_{3}$ are 0 with the corresponding eigenspace being spanned by $((1-\alpha), 0, \delta)$, and $-\delta<0$ with the corresponding eigenspace being spanned by $(1,0,0)$, as well as

$$
\beta_{2} \cdot h_{i}-\delta \begin{cases}<0, & \text { for } h_{i}<h^{*}, \\ =0, & \text { for } h_{i}=h^{*}:=\delta \cdot \beta_{2}^{-1}=\alpha \cdot \beta_{3} \cdot \beta_{2}^{-1}, \\ >0, & \text { for } h_{i}>h^{*},\end{cases}
$$

with the corresponding eigenspace being spanned by $\left(\beta_{1} \cdot f_{0}, \beta_{2} \cdot h_{i}, 0\right)$. Hence, the elements of $\Gamma_{3}$ are Lyapunov stable equilibrium points for $0 \leq h_{i}<h^{*}$ and unstable ones for $h^{*} \leq h_{i}$.

Finally, below the bifurcation point, i.e. for $\alpha \cdot \beta_{3}<\delta$, there is an additional equilibrium point $x^{*}:=\left(f^{*}, m^{*}, h^{*}\right)$ in the interior of $\mathscr{P}_{0}^{+}$: From Eq. (4.2) we first obtain $h^{*}:=\delta \cdot \beta_{2}^{-1}$. Plugging this into Eq. (4.3) leads to

$$
\beta_{1} \cdot f \cdot m=\frac{\delta \cdot\left(\delta-\alpha \cdot \beta_{3}\right)}{\beta_{2} \cdot(1-\gamma)}>0
$$

which requires $\alpha \cdot \beta_{3}<\delta$. Substituting $\beta_{1} \cdot f \cdot m$ accordingly into Eq. (4.1) gives

$$
f^{*}:=\frac{\gamma \cdot \beta_{1} \cdot\left(\delta-\alpha \cdot \beta_{3}\right)+(1-\gamma) \cdot(1-\alpha) \cdot \beta_{3}}{\beta_{2} \cdot(1-\gamma)}>0
$$

and

$$
m^{*}:=\frac{\delta \cdot\left(\delta-\alpha \cdot \beta_{3}\right)}{\gamma \cdot \beta_{1} \cdot\left(\delta-\alpha \cdot \beta_{3}\right)+(1-\gamma) \cdot(1-\alpha) \cdot \beta_{3}}>0
$$

The correspondingly transformed equivalent dynamics about $x^{*}:=\left(f^{*}, m^{*}, h^{*}\right)$ are given by

$$
\frac{\mathrm{d}}{\mathrm{d} t}\left(\begin{array}{c}
f \\
m \\
h
\end{array}\right)=\underbrace{\left(\begin{array}{ccc}
\gamma \cdot \beta_{1} \cdot m^{*}-\delta & \gamma \cdot \beta_{1} \cdot f^{*} & (1-\alpha) \cdot \beta_{3} \\
0 & 0 & \beta_{2} \cdot m^{*} \\
(1-\gamma) \cdot \beta_{1} \cdot m^{*} & (1-\gamma) \cdot \beta_{1} \cdot f^{*} & \alpha \cdot \beta_{3}-\delta
\end{array}\right)}_{=: B_{4}}\left(\begin{array}{c}
f \\
m \\
h
\end{array}\right)+\left(\begin{array}{c}
\gamma \cdot \beta_{1} \cdot f \cdot m \\
\beta_{2} \cdot h \cdot m \\
(1-\gamma) \cdot \beta_{1} \cdot f \cdot m
\end{array}\right) .
$$

Due to the complicated nature of $f^{*}$ and $m^{*}$ as well as the nearly complete occupation of $B_{4}$ with non-vanishing entries a direct computation of the eigenvalues and its corresponding eigenvectors is cumbersome. Therefore, we apply an indirect approach to determine the stability of the equilibrium $\left(f^{*}, m^{*}, h^{*}\right)$. Let $B_{4}:=\left(b_{i, j}\right)_{i, j=1,2,3}$, then the characteristic polynomial $\chi_{B_{4}}(\lambda)$ of $B_{4}$ reads

$$
\chi_{B_{4}}(\lambda)=-\lambda^{3}+\left(b_{1,1}+b_{3,3}\right) \cdot \lambda^{2}-\left(b_{1,1} \cdot b_{3,3}-b_{1,3} \cdot b_{3,1}\right) \cdot \lambda+b_{1,2} \cdot b_{2,3} \cdot b_{3,1} .
$$




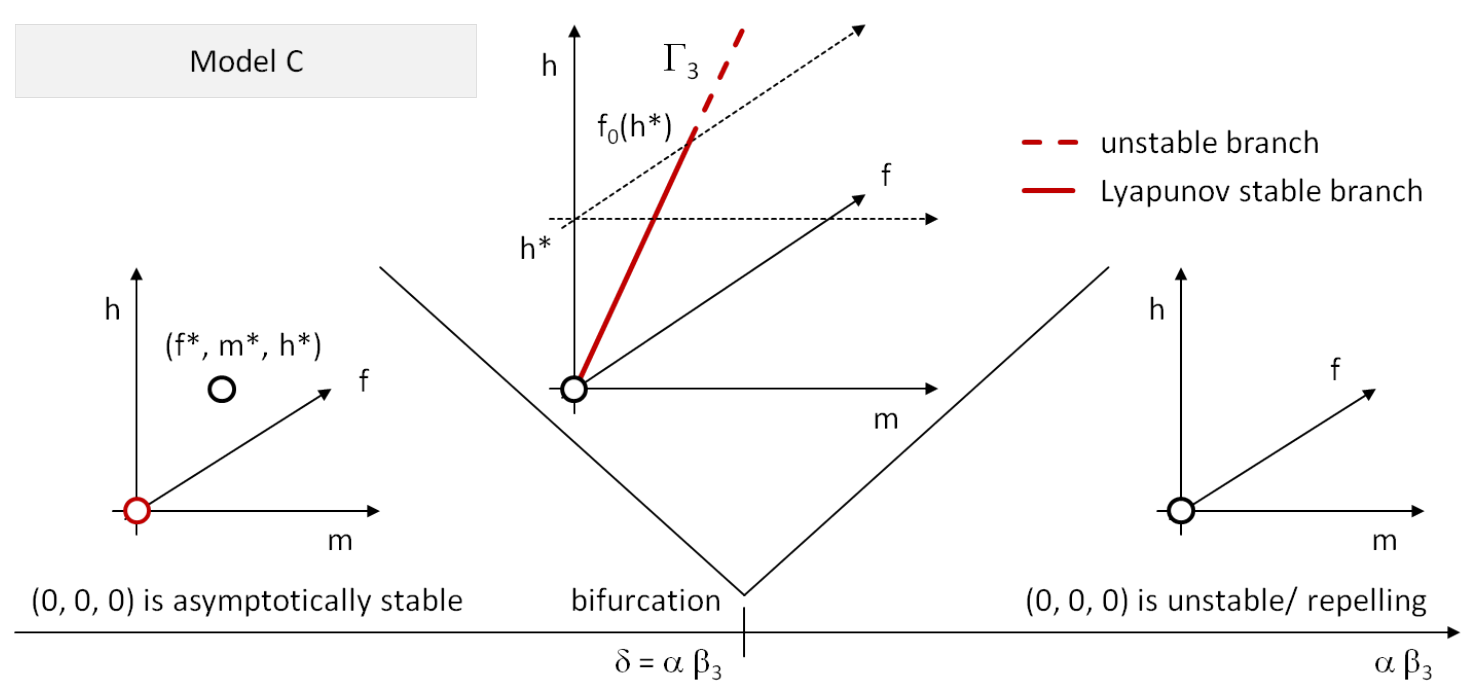

Figure 4.1: Sketch of the stability bifurcation of the extinction equilibrium at the origin at the bifurcation point $\alpha \cdot \beta_{3}^{-1}=\delta$, as well as of the transformation of the equilibrium $\left(f^{*}, m^{*}, h^{*}\right)$ with the occurrence of the line of equilibria $\Gamma_{3}$.

As $b_{1,1}=\gamma \cdot \beta_{1} \cdot m^{*}-\delta<0$ Descartes's sign rule gives that there is one positive real positive root, such that the equilibrium $\left(f^{*}, m^{*}, h^{*}\right)$ is unstable. For $\alpha \cdot \beta_{3} \uparrow \delta$ the $m$-component of the equilibrium $\left(f^{*}, m^{*}, h^{*}\right)$ vanishes such that the interior point $\left(f^{*}, m^{*}, h^{*}\right)$ moves with increasing values of $\alpha \cdot \beta_{3}$ towards the $f$-h-face of the first orthant, and eventually bifurcates to the line of equilibria $\Gamma_{3}$ at $\alpha \cdot \beta_{3}=\delta$.

The complete bifurcation diagram is sketched in Fig. 4.1. Note that no further equilibria of Eqs. (4.1)-(4.3) exist and we have thus indeed established a complete classification of the equilibria of our system including their stability.

In analogy to Model A we again have that the complete population survives/ proliferates for admissible parameter values $\delta<\alpha \beta_{3}$. In addition, model $\mathrm{C}$ offers some survival/ proliferation advantage at the bifurcation point.

\section{Model D}

Finally, we perform the same extension as from Model A to Model B and assume that the dynamics described by Model C are altered in view of a non-self-fertilizing hermaphrodite population. I.e., in particular the transport from female-male offspring towards the hermaphrodite sub-population is hence added. Assuming furthermore once again complete mixing and spatial homogeneity of the involved populations, then, according to the standard description of population dynamics, this leads to the following ordinary differential equation model D:

$$
\begin{aligned}
\dot{f} & =\gamma \cdot \beta_{1} \cdot f \cdot m+(1-\alpha) \cdot \beta_{3} \cdot h^{2}-\delta \cdot f, \\
\dot{m} & =\beta_{2} \cdot h \cdot m-\delta \cdot m, \\
\dot{h} & =\alpha \cdot \beta_{3} \cdot h^{2}+(1-\gamma) \cdot \beta_{1} \cdot f \cdot m-\delta \cdot h,
\end{aligned}
$$

Again, the non-negative orthant $\mathscr{P}_{0}^{+}$is invariant for all admissible values of the parameters, and, as in Model C, a vanishing hermaphrodite population does not automatically imply extinction of the complete population compared to Model B.

Though, in complete analogy to Model B, the origin $(f, m, h)=(0,0,0) \in \mathscr{P}_{0}^{+}$is an asymptotically stable equilibrium for all admissible parameter values (complete extinction equilibrium), as the local linearized dynamics about the origin are determined by

$$
\frac{\mathrm{d}}{\mathrm{d} t}\left(\begin{array}{l}
f \\
m \\
h
\end{array}\right)=\left(\begin{array}{ccc}
-\delta & 0 & 0 \\
0 & -\delta & 0 \\
0 & 0 & -\delta
\end{array}\right)\left(\begin{array}{l}
f \\
m \\
h
\end{array}\right)+\left(\begin{array}{c}
\gamma \cdot \beta_{1} \cdot f \cdot m+(1-\alpha) \cdot \beta_{3} \cdot h^{2} \\
\beta_{2} \cdot h \cdot m \\
(1-\gamma) \cdot \beta_{1} \cdot f \cdot m+\alpha \cdot \beta_{3} \cdot h^{2}
\end{array}\right) .
$$

Next, in complete analogy to Model $\mathrm{B}$, for all admissible values of the parameters the point $\left(f_{0}, 0, h_{0}\right)$ is an equilibrium as well, where $h_{0}:=\delta \cdot\left(\alpha \cdot \beta_{3}\right)^{-1}$ and $f_{0}:=\delta \cdot(1-\alpha) \cdot\left(\alpha^{2} \cdot \beta_{3}\right)^{-1}=(1-\alpha) \cdot \alpha^{-1} \cdot h_{0}$. The local linearized dynamics about $\left(f_{0}, 0, h_{0}\right)$ read as

$$
\frac{\mathrm{d}}{\mathrm{d} t}\left(\begin{array}{l}
f \\
m \\
h
\end{array}\right)=\underbrace{\left(\begin{array}{ccc}
-\delta & \gamma \cdot \beta_{1} \cdot f_{0} & 2 \cdot(1-\alpha) \cdot h_{0} \\
0 & \beta_{2} \cdot h_{0}-\delta & 0 \\
0 & (1-\gamma) \cdot \beta_{1} \cdot f_{0} & 2 \cdot \alpha \cdot \beta_{3} \cdot h_{0}-\delta
\end{array}\right)}_{=: B_{4}}\left(\begin{array}{c}
f \\
m \\
h
\end{array}\right)+\left(\begin{array}{c}
\gamma \cdot \beta_{1} \cdot f \cdot m+(1-\alpha) \cdot \beta_{3} \cdot h^{2} \\
\beta_{2} \cdot h \cdot m \\
(1-\gamma) \cdot \beta_{1} \cdot f \cdot m+\alpha \cdot \beta_{3} \cdot h^{2}
\end{array}\right),
$$

where the systems matrix $B_{4}$ has three distinct eigenvalues of algebraic multiplicity one: $-\delta<0,2 \cdot \alpha \cdot \beta_{3} \cdot h_{0}-\delta=\delta>0$, as well as $\beta_{2} \cdot h_{0}-\delta$ which is negative for $h_{0}<\delta \cdot \beta_{2}^{-1}$, vanishes for $h_{0}=h^{*}=\delta \cdot \beta_{2}^{-1}$, and is positive for $h_{0}<\delta \cdot \beta_{2}^{-1}$. Hence, the equilibrium $\left(f_{0}, 0, h_{0}\right)$ is unstable and the stability of one of its eigen-manifolds changes at $h_{0}=h^{*}$.

Finally, for parameter values with $\beta_{2}>\alpha \cdot \beta_{3}$, there is a further equilibrium point $\left(f^{*}, m^{*}, h^{*}\right)$ in the interior of $\mathscr{P}_{0}^{+}:$From Eq. (5.2) we get $h^{*}=\delta \cdot \beta_{2}^{-1}$, and plugging this into Eq. (5.2), we obtain

$$
\beta_{1} \cdot f \cdot m=\frac{\delta^{2} \cdot\left(\beta_{2}-\alpha \cdot \beta_{3}\right)}{\beta_{2}^{2} \cdot(1-\gamma)}>0,
$$


which requires $\beta_{2}>\alpha \cdot \beta_{3}$. Substituting $\beta_{1} \cdot f \cdot m$ accordingly into Eq. (5.1) gives

$$
f^{*}=\frac{\delta \cdot\left(\gamma \cdot\left(\beta_{2}-\alpha \cdot \beta_{3}\right)+(1-\alpha) \cdot(1-\gamma) \cdot \beta_{3}\right)}{\beta_{2}^{2} \cdot(1-\gamma)}>0,
$$

and

$$
m^{*}=\frac{\delta \cdot\left(\beta_{2}-\alpha \cdot \beta_{3}\right)}{\beta_{1} \cdot\left(\gamma \cdot\left(\beta_{2}-\alpha \cdot \beta_{3}\right)+(1-\alpha) \cdot(1-\gamma) \cdot \beta_{3}\right)}>0 .
$$

Due to $\beta_{2} \cdot h^{*}-\delta=0$, the correspondingly transformed equivalent dynamics about $\left(f^{*}, m^{*}, h^{*}\right)$ are given by

$$
\frac{\mathrm{d}}{\mathrm{d} t}\left(\begin{array}{c}
f \\
m \\
h
\end{array}\right)=\underbrace{\left(\begin{array}{ccc}
\gamma \cdot \beta_{1} \cdot m^{*}-\delta & \gamma \cdot \beta_{1} \cdot f^{*} & 2 \cdot(1-\alpha) \cdot h^{*} \\
0 & 0 & \beta_{2} \cdot m^{*} \\
(1-\gamma) \cdot \beta_{1} \cdot m^{*} & (1-\gamma) \cdot \beta_{1} \cdot f^{*} & 2 \cdot \alpha \cdot \beta_{3} \cdot h^{*}-\delta
\end{array}\right)}_{=: B_{5}}\left(\begin{array}{c}
f \\
m \\
h
\end{array}\right)+\left(\begin{array}{c}
\gamma \cdot \beta_{1} \cdot f \cdot m+(1-\alpha) \cdot \beta_{3} \cdot h^{2} \\
\beta_{2} \cdot h \cdot m \\
(1-\gamma) \cdot \beta_{1} \cdot f \cdot m+\alpha \cdot \beta_{3} \cdot h^{2}
\end{array}\right)
$$

such that we can follow the same line of argumentation as in Model C. Let $B_{5}:=\left(b_{i, j}\right)_{i, j=1,2,3}$, then its characteristic polynomial $\chi_{B_{4}}(\lambda)$ reads

$$
\chi_{B_{5}}(\lambda)=-\lambda^{3}+\left(b_{1,1}+b_{3,3}\right) \cdot \lambda^{2}-\left(b_{1,1} \cdot b_{3,3}-b_{1,3} \cdot b_{3,1}\right) \cdot \lambda+b_{1,2} \cdot b_{2,3} \cdot b_{3,1} .
$$

In complete analogy to Model C, we have that $b_{1,1}=\gamma \cdot \beta_{1} \cdot m^{*}-\delta<0$, and hence Descartes's sign rule implies that there is one positive real positive root, such that the equilibrium $\left(f^{*}, m^{*}, h^{*}\right)$ is unstable. For $\alpha \cdot \beta_{3} \uparrow \beta_{2}$ the $m$-component of the equilibrium $\left(f^{*}, m^{*}, h^{*}\right)$ vanishes such that the interior point $\left(f^{*}, m^{*}, h^{*}\right)$ moves with increasing values of $\alpha \cdot \beta_{3}$ towards the $f-h$-face of the first orthant, and eventually coincides with the previously discussed equilibrium $\left(f^{*}=f_{0}, 0, h^{*}=h_{0}\right)$ at $\alpha \cdot \beta_{3}=\beta_{2}$.

\section{Resume}

During the course of our mathematical discussion and comparison of the two models we can conclude, that models A and D show a survival/ proliferation advantage over models B and C, respectively, for small population numbers. Survival/ proliferation in models A and D depends only on the birth and death parameters of the hermaphrodite populations and not on certain threshold populations. In models B and D the origin (complete extinction equilibrium) is always asymptotically stable and hence supporting the extinction of the complete population, whereas models A and prevent, under some further conditions, the complete extinction due to the unstable/ repulsive nature of the origin.

Additionally, to numerical studies already carried out in the literature, cf. Ref. [16], we analytically showed the extremely robust survival/ proliferation properties of self-fertilizing species. Moreover, in contrast, to the usual heterogametic view of reproduction that predict convergence towards a bisexual species, cf. Refs. [14, 15], the homogametic reproduction strategy used by Aunema Rhodensis shows clear advantages in terms of a survival of all sub-species.

Thus, the mathematical discussion of the dynamics of self-fertilizing trisexual homogametic species adds a further piece to the puzzle of evolutionary competitiveness.

\section{Appendix A: Mathematical Models for Species with Neither Hermaphrodites-Producing Females Nor Females-Producing Hermaphrodites}

As discussed in the introduction, the two mathematical models in this appendix serve a reference and a completion of our study of the dynamics of trisexual species. They are rather simple in the mutual interaction of the female, male and hermaphrodite populations and their mathematical analysis is straight-forward utilizing the same lines of argumentation as applied in the main part of the article.

\section{Model $\mathrm{E}_{1}$ : A Species with Self-Fertilizing Hermaphrodites}

A mathematical model of a trisexual species with self-fertilizing hermaphrodites, like our Auanema Rhodensis, where there are nohermaphrodites producing females and no-females producing hermaphrodites can be considered as a special limit case of Model A with $\alpha=1$ as

$$
\dot{f}=\left(\beta_{1} \cdot m-\delta\right) \cdot f, \quad \dot{m}=\left(\beta_{2} \cdot h-\delta\right) \cdot m, \quad \text { and } \quad \dot{h}=\left(\beta_{3}-\delta\right) \cdot h,
$$

where, as previously, $\beta_{1}, \beta_{2}, \beta_{3}>0$ are the proportionality factors related to birth events, and $\delta>0$ is the sex-independent proportionality factor related to death events. We will call this set of equations together with the corresponding initial conditions $f(0)=f_{i} \geq 0, m(0)=m_{i} \geq 0$ and $h(0)=h_{i} \geq 0$ as Model $\mathrm{E}_{1}$.

The non-negative orthant $\mathscr{P}_{0}^{+}$is invariant under the dynamics of Model $\mathrm{E}_{1}$. Immediately, we see that the origin $(0,0,0)$ is an equilibrium point for all admissible values of the parameters, that is asymptotically stable for $\beta_{3}<\delta$, Lyapunov stable for $\beta_{3}=\delta$, and unstable for $\beta_{3}>\delta$. As in Model A, we have a bifurcation point at $\beta_{3}=\delta$.

In particular, for $\beta_{3}<\delta$ (or $\beta_{3}>\delta$ ) we have that $\dot{h}<0$ (or $\dot{h}>0$ ) for all times such that the hermaphrodite population eventually vanishes (or grows beyond all bounds). This implies, that at some finite time $\beta_{2} \cdot h-\delta<0$ (or $\beta_{2} \cdot h-\delta>0$ ) such that the male population eventually vanishes (or grows beyond all bounds). Consequently, at some finite time $\beta_{1} \cdot m-\delta<0$ (or $\beta_{1} \cdot m-\delta>0$ ) such that the female population eventually vanishes (or grows beyond all bounds). Hence, for $\beta_{3}<\delta$ (or $\beta_{3}>\delta$ ) the origin is a globally stable (or globally unstable) equilibrium. Note, that there are no further equilibrium points for $\beta_{3} \neq \delta$.

Finally, the dynamics for $\beta_{3}=\delta$ are sketched in Fig. 6.1 and a complete picture of the additional equilibria that occur at this set of parameters is given in the following proposition. 

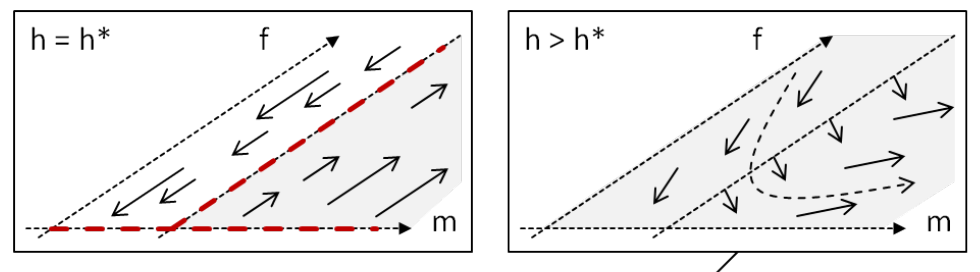

Model $\mathrm{E}_{1}$
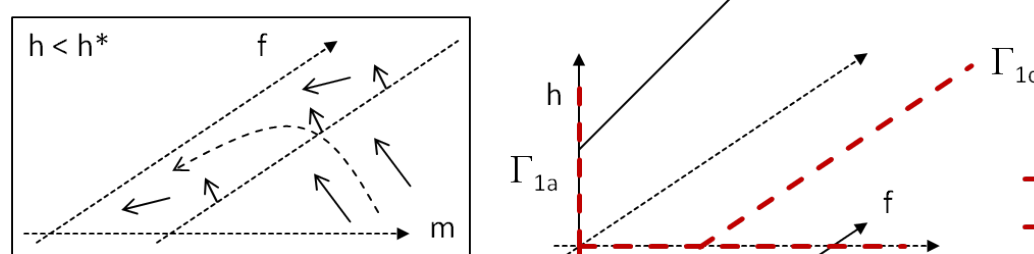

- unstable branch

— Lyapunov stable branch

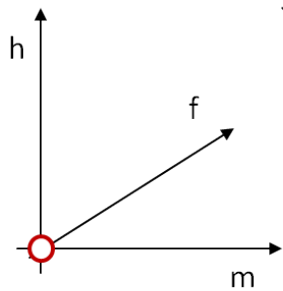

$h^{*}$
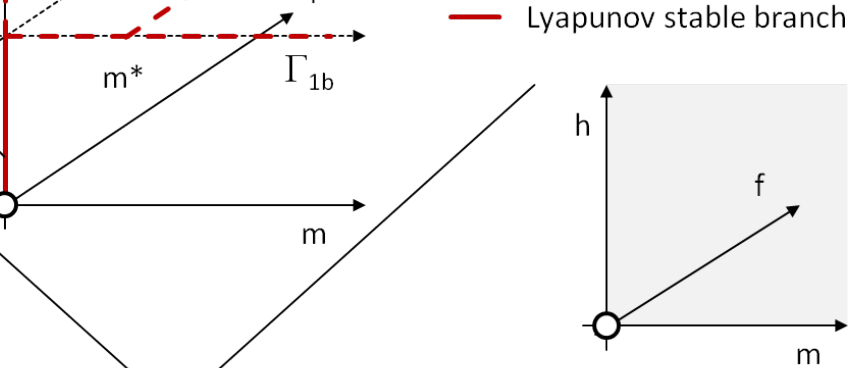

$(0,0,0)$ is asymptotically stable

bifurcation

$\delta=\beta_{3}$

$(0,0,0)$ is unstable/ repelling

Figure 6.1: Sketch of the bifurcation scenario and some illustrative dynamics of Model $\mathrm{E}_{1}$.

Theorem E.1: Additional Lines of Equilibria at the Bifurcation Point. Let $h^{*}:=\delta \cdot \beta_{2}^{-1}$ and $m^{*}:=\delta \cdot \beta_{1}^{-1}$. For $\beta_{3}=\delta$ and besides the origin there are three lines of equilibria

- $\Gamma_{1 a}:=\left\{\left(0,0, h_{i}\right) \in \mathscr{P}_{0}^{+}: h_{i} \geq 0\right\}$, where each element is Lyapunov stable for $0 \leq h_{i} \leq h^{*}$ and unstable for $h^{*}<h_{i}$,

- $\Gamma_{1 b}:=\left\{\left(0, m_{i}, h^{*}\right) \in \mathscr{P}_{0}^{+}: m_{i} \geq 0\right\}$, where each element is unstable, and

- $\Gamma_{1 c}:=\left\{\left(f_{i}, m^{*}, h^{*}\right) \in \mathscr{P}_{0}^{+}: f_{i} \geq 0\right\}$, where each element is unstable.

Moreover, there are no further equilibrium points for $\beta_{3}=\delta$.

Proof: A short calculation leads to the existence of these lines of equilibria and that, besides the origin, there are no further ones. The local dynamics about each equilibrium point $(\tilde{f}, \tilde{m}, \tilde{h})$ are given by

$$
\frac{\mathrm{d}}{\mathrm{d} t}\left(\begin{array}{c}
f \\
m \\
h
\end{array}\right)=\underbrace{\left(\begin{array}{ccc}
\beta_{1} \cdot \tilde{m}-\delta & \beta_{1} \cdot \tilde{f} & 0 \\
0 & \beta_{2} \cdot \tilde{h}-\delta & \beta_{2} \cdot \tilde{m} \\
0 & 0 & 0
\end{array}\right)}_{=: E_{1}(\tilde{f}, \tilde{m}, \tilde{h})}\left(\begin{array}{c}
f \\
m \\
h
\end{array}\right)+\left(\begin{array}{c}
\beta_{1} \cdot f \cdot m \\
\beta_{2} \cdot h \cdot m \\
0
\end{array}\right) .
$$

The eigenvalues of $E_{1}\left(0,0, h_{i}\right)$, with a specific value $h_{i} \geq 0$, are $\lambda_{1}:=0$ with the corresponding eigenspace being spanned by $(0,0,1)$, $\lambda_{2}:=-\delta<0$ with the corresponding eigenspace being spanned by $(1,0,0)$, and $\lambda_{3}:=\beta_{2} \cdot h_{i}-\delta$ with the corresponding eigenspace being spanned by $(0,1,0)$. In particular, $\lambda_{3}<0$ if $0 \leq h_{i}<h^{*}, \lambda_{3}=0$ if $h_{i}=h^{*}$, and $\lambda_{3}>0$ if $h^{*}<h_{i}$.

The eigenvalues of $E_{1}\left(0, m_{i}, h^{*}\right)$, with a specific value $m_{i} \geq 0$, are $\lambda_{1}:=0$ with algebraic multiplicity two and the corresponding onedimensional eigenspace being spanned by $(0,1,0)$, and $\lambda_{2}:=\beta_{1} \cdot m_{i}-\delta$ with the corresponding eigenspace being spanned by $(1,0,0)$. In particular, $\lambda_{2}<0$ if $0 \leq m_{i}<m^{*}, \lambda_{2}=0$ if $m_{i}=m^{*}$, and $\lambda_{2}>0$ if $m^{*}<m_{i}$.

The only eigenvalue of $E_{1}\left(f_{i}, m^{*}, h^{*}\right)$, with a specific value of $f_{i} \geq 0$, is $\lambda_{1}:=0$ with algebraic multiplicity three and corresponding one-dimensional eigenspace being spanned by $(1,0,0)$.

The global dynamics $\beta_{3}=\delta$ are derived in complete analogy to Model A.

\section{Model $\mathrm{E}_{2}$ : A Species with Non-Self-Fertilizing Hermaphrodites}

Next, a mathematical model of a trisexual species with non-self-fertilizing hermaphrodites, where there are no-hermaphrodites-producing females and no-females producing hermaphrodites can be considered as a special limit case of Model B with $\alpha=1$ as

$$
\dot{f}=\left(\beta_{1} \cdot m-\delta\right) \cdot f, \quad \dot{m}=\left(\beta_{2} \cdot h-\delta\right) \cdot m, \quad \text { and } \quad \dot{h}=\left(\beta_{3} \cdot h-\delta\right) \cdot h,
$$

where, as previously, $\beta_{1}, \beta_{2}, \beta_{3}>0$ are the proportionality factors related to birth events, and $\delta>0$ is the sex-independent proportionality factor related to death events. We will call this set of equations together with the corresponding initial conditions $f(0)=f_{i} \geq 0, m(0)=m_{i} \geq 0$ and $h(0)=h_{i} \geq 0$ as Model $\mathrm{E}_{2}$. 

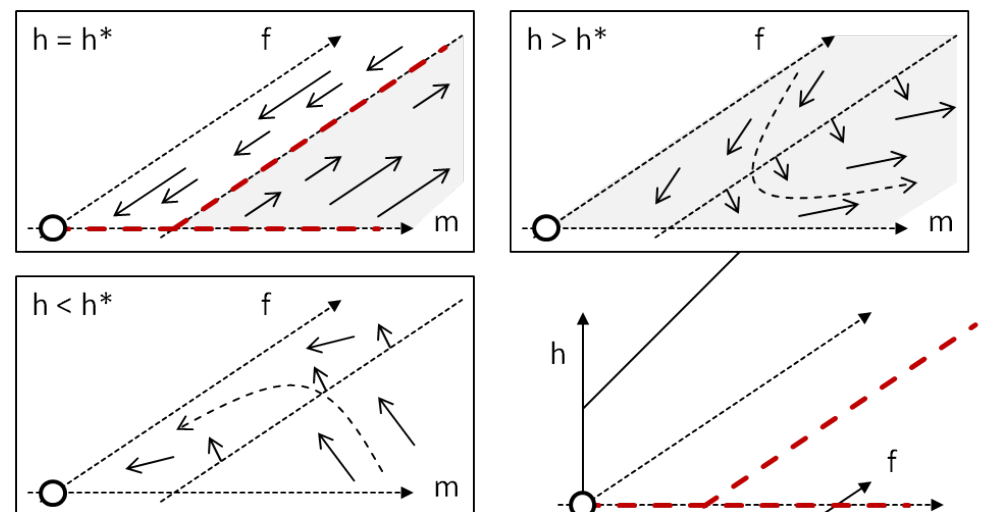

.

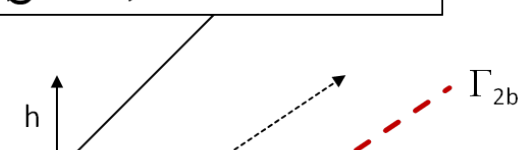

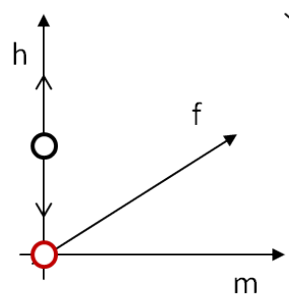

$(0,0,0)$ is asymptotically stable

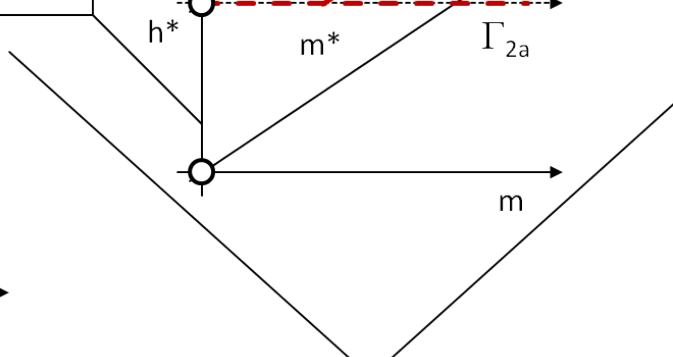

bifurcation
Model $\mathrm{E}_{2}$

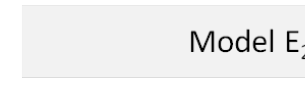

- - unstable branch

— Lyapunov stable branch

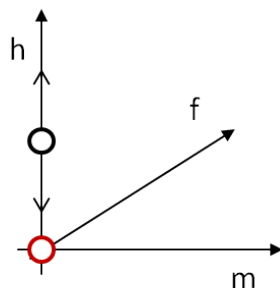

$(0,0,0)$ is asymptotically stable

$\beta_{2}=\beta_{3}$

Figure 6.2: Sketch of the bifurcation scenario and some illustrative dynamics of Model $\mathrm{E}_{2}$.

The non-negative orthant $\mathscr{P}_{0}^{+}$is invariant under the dynamics of Model $\mathrm{E}_{1}$, and for all values of the parameters the origin $(0,0,0)$ is an asymptotically stable equilibrium point with $\operatorname{diag}(-\delta,-\delta,-\delta)$ as the matrix of linearization about the origin. Moreover, for all values of the parameters, the point $\left(0,0, h^{*}\right)$, with $h^{*}:=\delta \cdot \beta_{3}^{-1}$, is a further equilibrium point. The matrix of linearization about $\left(0,0, h^{*}\right)$ is $\operatorname{diag}\left(-\delta, \delta \cdot\left(\beta_{2} \cdot \beta_{3}^{-1}-1\right), \delta\right)$ such that this equilibrium is unstable though exhibits a change of stability at $\beta_{2}=\beta_{1}$ where a geometrically two-dimensional (asymptotically) stable sub-space bifurcates into a (asymptotically) stable one and an unstable one.

Finally, the dynamics for Model $\mathrm{E}_{2}$ are sketched in Fig. 6.2 and a complete picture of the additional equilibria that occur at $\beta_{3}=\beta_{2}$ is given in the following proposition.

Theorem E.2: Additional Lines of Equilibria. Let $h^{*}:=\delta \cdot \beta_{3}^{-1}$ and $m^{*}:=\delta \cdot \beta_{1}^{-1}$. For $\beta_{3}=\beta_{2}$ and besides the origin there are two lines of equilibria

- $\Gamma_{2 a}:=\left\{\left(0, m_{i}, h^{*}\right) \in \mathscr{P}_{0}^{+}: m_{i} \geq 0\right\}$, where each element is unstable, and

- $\Gamma_{2 b}:=\left\{\left(f_{i}, m^{*}, h^{*}\right) \in \mathscr{P}_{0}^{+}: f_{i} \geq 0\right\}$, where each element is unstable.

Moreover, there are no further equilibrium points for $\beta_{3}=\beta_{2}$.

Proof: A short calculation leads to the existence of these lines of equilibria and that, besides the origin, there are no further ones. The local dynamics about each equilibrium point $(\tilde{f}, \tilde{m}, \tilde{h})$ are given by

$$
\frac{\mathrm{d}}{\mathrm{d} t}\left(\begin{array}{c}
f \\
m \\
h
\end{array}\right)=\underbrace{\left(\begin{array}{ccc}
\beta_{1} \cdot \tilde{m}-\delta & \beta_{1} \cdot \tilde{f} & 0 \\
0 & 0 & \beta_{2} \cdot \tilde{m} \\
0 & 0 & 0
\end{array}\right)}_{=: E_{2}(\tilde{f}, \tilde{m}, \tilde{h})}\left(\begin{array}{c}
f \\
m \\
h
\end{array}\right)+\left(\begin{array}{c}
\beta_{1} \cdot f \cdot m \\
\beta_{2} \cdot h \cdot m \\
\beta_{3} \cdot h^{2}
\end{array}\right) .
$$

The eigenvalues of $E_{2}\left(0, m_{i}, h^{*}\right)$, with a specific value $m_{i} \geq 0$, are $\lambda_{1}:=0$ with algebraic multiplicity two and the corresponding onedimensional eigenspace being spanned by $(0,1,0)$, and $\lambda_{2}:=\beta_{1} \cdot m_{i}-\delta$ with the corresponding eigenspace being spanned by $(1,0,0)$. In particular, $\lambda_{2}<0$ if $0 \leq m_{i}<m^{*}, \lambda_{2}=0$ if $m_{i}=m^{*}$, and $\lambda_{2}>0$ if $m^{*}<m_{i}$.

The only eigenvalue of $E_{2}\left(f_{i}, m^{*}, h^{*}\right)$, with a specific value of $f_{i} \geq 0$, is $\lambda_{1}:=0$ with algebraic multiplicity three and corresponding one-dimensional eigenspace being spanned by $(1,0,0)$.

The global dynamics $\beta_{3}=\delta$ are derived in complete analogy to Model B.

\section{Appendix B: Mathematical Models for Species with Hermaphrodites-Producing Females and No- Females-Producing Hermaphrodites}

The two rather simple mathematical models in this appendix close the remaining gap in our discussion by studying species with hermaphrodites-producing females and no-females-producing hermaphrodites, i.e. models where female XX-offspring can evolve to 
both females and hermaphrodites, whereas hermaphrodite XX-offspring can only evolve to hermaphrodites.

\section{Model $F_{1}$ : A Species with Self-Fertilizing Hermaphrodites}

Analogue to our previous modeling the driving biological dynamics in the setting with self-fertilizing hermaphrodites, like for Auanema Rhodensis, with hermaphrodites-producing females and no-females-producing hermaphrodites read as

$$
\dot{f}=\left(\gamma \cdot \beta_{1} \cdot m-\delta\right) \cdot f, \quad \text { and } \quad \dot{m}=\left(\beta_{2} \cdot h-\delta\right) \cdot m,
$$

as well as

$$
\dot{h}=\beta_{3} \cdot h+(1-\gamma) \cdot \beta_{1} \cdot m \cdot f-\delta \cdot h,
$$

where $\beta_{1}, \beta_{2}, \beta_{3}>0$ are proportionality factors related to birth events, $\delta>0$ is the sex-independent proportionality factor related to death events, and $\gamma$ the transport factor from female offspring towards the female sub-population (correspondingly, the factor $1-\gamma$ is the transport factor from female offspring towards the hermaphrodites sub-population). We will call this set of equations together with the corresponding initial conditions $f(0)=f_{i} \geq 0, m(0)=m_{i} \geq 0$ and $h(0)=h_{i} \geq 0$ as Model $\mathrm{F}_{1}$.

The non-negative orthant $\mathscr{P}_{0}^{+}$is invariant under the dynamics of Model $\mathrm{F}_{1}$, and for all admissible values of the parameters the origin $(0,0,0)$ is an equilibrium with $\operatorname{diag}\left(-\delta,-\delta, \beta_{3}-\delta\right)$ as the matrix of linearization about it. In particular, the origin is asymptotically stable for $\beta_{3}<\delta$, Lyapunov stable for $\beta_{3}=\delta$, and unstable for $\beta_{3}>\delta$. As in Model A, we have a bifurcation point at $\beta_{3}=\delta$.

Let $h^{*}:=\delta \cdot \beta_{2}^{-1}$. At $\beta_{3}=\delta$ we have two additional lines of equilibria:

- $\Gamma_{3, a}:=\left\{\left(0,0, h_{i}\right) \in \mathscr{P}_{0}^{+}: h_{i} \geq 0\right\}$, where, analogous to the proof of Theorem E.1, each element is Lyapunov stable for $0 \leq h_{i} \leq h^{*}$ and unstable for $h^{*}<h_{i}$, and

- $\Gamma_{3, b}:=\left\{\left(0, m_{i}, h^{*}\right) \in \mathscr{P}_{0}^{+}: m_{i} \geq 0\right\}$, where, analogous to the proof of Theorem E.1, each element is unstable.

If $\beta_{3}>\delta$ holds, besides the origin, another equilibrium exists at the point $(f, m, h):=\left(f^{*}, m^{*}, h^{*}\right)$, where $m^{*}:=\delta \cdot\left(\gamma \cdot \beta_{1}\right)^{-1}, h^{*}:=\delta \cdot \beta_{2}^{-1}$, and

$$
f^{*}:=\frac{\left(\beta_{3}-\delta\right) \cdot h^{*}}{(1-\gamma) \cdot \beta_{1} \cdot m^{*}}=\frac{\left(\beta_{3}-\delta\right) \cdot \gamma}{(1-\gamma) \cdot \beta_{2}}>0
$$

The correspondingly transformed equivalent dynamics about this interior mixed species equilibrium $\left(f^{*}, m^{*}, h^{*}\right)$ are given by

$$
\frac{\mathrm{d}}{\mathrm{d} t}\left(\begin{array}{c}
f \\
m \\
h
\end{array}\right)=\underbrace{\left(\begin{array}{ccc}
\overbrace{\gamma \cdot \beta_{1} \cdot m^{*}-\delta}^{0} & \gamma \cdot \beta_{1} \cdot f^{*} & 0 \\
0 & 0 & \beta_{2} \cdot m^{*} \\
(1-\gamma) \cdot \beta_{1} \cdot m^{*} & (1-\gamma) \cdot \beta_{1} \cdot f^{*} & \beta_{3}-\delta
\end{array}\right)}_{=: F_{1}\left(f^{*}, m^{*}, h^{*}\right)}\left(\begin{array}{c}
f \\
m \\
h
\end{array}\right)+\left(\begin{array}{c}
\gamma \cdot \beta_{1} \cdot f \cdot m \\
\beta_{2} \cdot h \cdot m \\
(1-\gamma) \cdot \beta_{1} \cdot f \cdot m
\end{array}\right) .
$$

The three distinct eigenvalues of $F_{1}\left(f^{*}, m^{*}, h^{*}\right)$, each with geometric multiplicity 1 , are $\lambda_{1}:=\delta \cdot \beta_{2} \cdot\left(\gamma \cdot \beta_{1}\right)^{-1}>0, \lambda_{2}:=\left(\beta_{3}-\delta\right) \cdot \gamma^{2}$. $\beta_{1}\left((1-\gamma) \cdot \beta_{2}\right)^{-1}$, and $\lambda_{3}=\delta \cdot(1-\gamma) \cdot \gamma^{-1}>0$. Hence, the equilibrium $\left(f^{*}, m^{*}, h^{*}\right)$ is unstable for all admissible parameter values.

Moreover, there are no further equilibrium points, and the global dynamics in Model $\mathrm{F}_{1}$ are derived in complete analogy to Model A.

\section{Model $F_{2}$ : A Species with Non-Self-Fertilizing Hermaphrodites}

The driving biological dynamics in the setting with non-self-fertilizing hermaphrodites together with hermaphrodites-producing females and no-females-producing hermaphrodites read as

$$
\dot{f}=\left(\gamma \cdot \beta_{1} \cdot m-\delta\right) \cdot f, \quad \text { and } \quad \dot{m}=\left(\beta_{2} \cdot h-\delta\right) \cdot m,
$$

as well as

$$
\dot{h}=\beta_{3} \cdot h^{2}+(1-\gamma) \cdot \beta_{1} \cdot m \cdot f-\delta \cdot h,
$$

where $\beta_{1}, \beta_{2}, \beta_{3}>0$ are proportionality factors related to birth events, $\delta>0$ is the sex-independent proportionality factor related to death events, and $\gamma$ the transport factor from female offspring towards the female sub-population (correspondingly, the factor $1-\gamma$ is the transport factor from female offspring towards the hermaphrodites sub-population). We will call this set of equations together with the corresponding initial conditions $f(0)=f_{i} \geq 0, m(0)=m_{i} \geq 0$ and $h(0)=h_{i} \geq 0$ as Model $\mathrm{F}_{2}$.

The non-negative orthant $\mathscr{P}_{0}^{+}$is invariant under the dynamics of Model $\mathrm{F}_{2}$, and for all admissible values of the parameters the origin $(0,0,0)$ is an asymptotically stable equilibrium with $\operatorname{diag}(-\delta,-\delta,-\delta)$ as the matrix of linearization about it.

Let $m^{*}:=\delta \cdot\left(\gamma \cdot \beta_{1}\right)^{-1}, h^{*}:=\delta \cdot \beta_{2}^{-1}$. For $0<\beta_{3}<\beta_{2}$, the point $\left(f^{*}, m^{*}, h^{*}\right)$ is an additional mixed species equilibium, where

$$
f^{*}=\frac{\left(\beta_{2}-\beta_{3}\right) \cdot \gamma \cdot \delta}{(1-\gamma) \cdot \beta_{2}^{2}}>0 .
$$

The correspondingly transformed equivalent dynamics about this interior mixed species equilibrium $\left(f^{*}, m^{*}, h^{*}\right)$ are given by

$$
\frac{\mathrm{d}}{\mathrm{d} t}\left(\begin{array}{c}
f \\
m \\
h
\end{array}\right)=\underbrace{\left(\begin{array}{ccc}
\overbrace{\gamma \cdot \beta_{1} \cdot m^{*}-\delta}^{=0} & \gamma \cdot \beta_{1} \cdot f^{*} & 0 \\
0 & 0 & \beta_{2} \cdot m^{*} \\
(1-\gamma) \cdot \beta_{1} \cdot m^{*} & (1-\gamma) \cdot \beta_{1} \cdot f^{*} & 2 \cdot \beta_{3} \cdot h^{*}-\delta
\end{array}\right)}_{=: F_{2}\left(f^{*}, m^{*}, h^{*}\right)}\left(\begin{array}{c}
f \\
m \\
h
\end{array}\right)+\left(\begin{array}{c}
\gamma \cdot \beta_{1} \cdot f \cdot m \\
\beta_{2} \cdot h \cdot m \\
(1-\gamma) \cdot \beta_{1} \cdot f \cdot m+\beta_{3} \cdot h^{2}
\end{array}\right),
$$


such that, in complete analogy to Model $\mathrm{F}_{1}$, this is an unstable equilibrium.

At $\beta_{3}=\beta_{2}$, additional to the origin, this mixed species equilibrium bifurcates to a line of equilibria $\Gamma_{4, a}:=\left\{\left(0, m_{i}, h^{*}\right) \in \mathscr{P}_{0}^{+}: m_{i}>0\right\}$ such that analogous to the proof of Theorem E.2 each member of this line is unstable.

Moreover, there are no further equilibrium points, and the global dynamics in Model $\mathrm{F}_{2}$ are derived in complete analogy to Model A.

\section{References}

[1] J. Chaudhuri, V. Kache, A. Pires-daSilva, Regulation of sexual plasticity in a nematode that produces males, females, and hermaphrodites, Current Biology, 21 (2011), 1548-1551,

[2] T. Hale, This Trisexual Worm Bends the Rules of Typical Genetics, IFL Science, 19/ 01/ 2018 (2018), http://www.iflscience.com/ plants-and-animals/a-trisexual-worm-bends-the-rules-of-typical-genetics/.

[3] D.C. Shakes, B.J. Neva, H. Huynh, J. Chaudhuri, A. Pires-daSilva, Asymmetric spermatocyte division as a mechanism for controlling sex ratios, Nature Communications, 2(157) (2011).

[4] S. Tandonnet, M.C. Farrell, G.D. Koutsovoulos, M.L. Blaxter, M. Parihar, P.L. Sadler, D.C. Shakes, A. Pires-daSilva, Sex- and gamete-specific patterns of X chromosome segregation in a trioecious nematode, Current Biology, 28 (2018), 93-99.

[5] N. Kanzaki, K. Kiontke, R. Tanaka, Y. Hirooka, A. Schwarz, T. Müller-Reichert, J. Chaudhuri, A. Pires-daSilva, Description of two three-gendered nematode species in the new genus Auanema (Rhabditina) that are models for reproductive mode evolution, Scientific Reports, 7 (2017), 11135.

[6] E. Garibaldi, M. Sobottka, A nonsmooth two-sex population model, Mathematical Biosciences, 253 (2014), 1-10.

[7] K.P. Hadeler, R. Waldstätter, A. Wörz-Busekros, Models for pair formation in bisexual populations, J. Math. Biol., 26 (1988), 635-649.

[8] D.G. Kendall, Stochastic processes and population growth, J. R. Stat. Soc. Ser. B Stat. Methodol., 11 (1949), $230-264$.

[9] D.J. Rankin, H. Kokko, Do males matter? The role of males in population dynamics, Oikos, 116 (2007), $335-348$.

[10] J.D. Murray, Mathematical Biology I. An Introduction, 3rd ed., Springer, 2002.

[11] J.D. Murray, Mathematical Biology II. Spatial Models and Biomedical Applications, 3rd ed., Springer, 2003.

[12] F. Rupp, J. Scheurle, Analysis of a Mathematical Model for Jellyfish Blooms and the Cambric Fish Invasion, Dynamical Systems and Differential Equations, DCDS Supplement 2013 Proceedings of the 9th AIMS International Conference (Orlando, USA) (2013), 663-672.

[13] F. Rupp, J. Scheurle, The dynamics of the jellyfish Joyride: Mathematical discussion of the causes to blooming, Math. Methods Appl. Sci., 38(16) (2015), 3408-3420.

[14] E.C. Buehler, S. Das, J.F. Cully Jr., Equilibrium and extinction in a trisexual diploid mating system: An investigation, K. Deb (editor), Genetic and Evolutionary Computation - GECCO 2004, Lecture Notes in Computer Science, vol 3102, Springer, Berlin, Heidelberg, 2004.

[15] E.C. Buehler, S. Das, J.F. Cully Jr., Equilibrium and Extinction in a Trisexual Diploid Mating System, 2004.

[16] K. Jaffe, The dynamics of the evolution of sex: Why the sexes are, in fact, always two?, Interciencia, 21(6) (1996), $259-267$. 